\title{
Credibility-Limited Base Revision: New Classes and Their Characterizations
}

\author{
Marco Garapa \\ Faculdade de Ciências Exatas e da Engenharia, \\ Universidade da Madeira, Campus Universitário da Penteada, \\ 9020-105 Funchal, Portugal \\ CIMA - Centro de Investigação em Matemática e Aplicações
}

MGARAPA@STAFF.UMA.PT

Eduardo Fermé

FERME@UMA.PT

Faculdade de Ciências Exatas e da Engenharia,

Universidade da Madeira, Campus Universitário da Penteada, 9020-105 Funchal, Portugal

NOVA Laboratory for Computer Science and Informatics (NOVA LINCS)

Maurício D. L. Reis

M_REIS@STAFF.UMA.PT

Faculdade de Ciências Exatas e da Engenharia,

Universidade da Madeira, Campus Universitário da Penteada,

9020-105 Funchal, Portugal

CIMA - Centro de Investigação em Matemática e Aplicações

\begin{abstract}
In this paper we study a kind of operator - known as credibility-limited base revisionswhich addresses two of the main issues that have been pointed out to the AGM model of belief change. Indeed, on the one hand, these operators are defined on belief bases (rather than belief sets) and, on the other hand, they are constructed with the underlying idea that not all new information is accepted. We propose twenty different classes of credibilitylimited base revision operators and obtain axiomatic characterizations for each of them. Additionally we thoroughly investigate the interrelations (in the sense of inclusion) among all those classes. More precisely, we analyse whether each one of those classes is or is not (strictly) contained in each of the remaining ones.
\end{abstract}

\section{Introduction}

Belief change became a major subject in artificial intelligence in the middle of the 1980's. The one that is nowadays considered the standard model of belief change is the AGM model, proposed by Alchourrón et al. (1985). In the AGM framework the belief state of an agent is represented by a belief set, i.e. a logically closed set of propositional sentences - which represents the beliefs of that agent. In the AGM model three kinds of change operators for belief sets are considered, namely:

- Expansions, whose output is a belief set which (may be inconsistent and) contains all the sentences of the original belief set and also the (sentence representing the) new information. 
- Contractions, whose output is a belief set that is a subset of the original one, which does not contain the sentence received as input, but contains as many as possible of the previous beliefs.

- Revisions, ${ }^{1}$ whose output is, whenever possible, a consistent belief set that contains the new belief and as many as possible of the previous beliefs.

The AGM model inspired many researchers to propose extensions and generalizations (for an overview see Fermé \& Hansson, 2011; and Fermé \& Hansson, 2018), from among which we highlight the following ones:

(a) Models of belief base change: These are models in which the belief state of an agent is represented by a set of sentences that is not, except as a limiting case, closed under logical consequence. These sets are called belief bases and have a fundamental property: they allow to distinguish between explicit beliefs, which are elements of the belief base, and derived beliefs, i.e., elements that are logical consequences of the belief base, but that are not explicitly present in the belief base. Belief bases are more suitable than belief sets for representing the belief states of real cognitive agents since, as it was pointed out by Gärdenfors and Rott (1995), "when we perform revisions or contractions, it seems that we never do it to the belief set itself $(\ldots)$ but rather on some typically finite base for the belief set". Additionally, because belief sets are often too big, eventually even infinite, they are not adequate for computational implementations of belief change models. The use of belief bases has been largely studied in the literature (Dalal, 1988; Fermé, 1992; Fuhrmann, 1988, 1991; Garapa, 2017; Hansson, 1989, 1991a, 1992b, 1994b; Nebel, 1989; Rott, 2000; Wassermann, 2000).

(b) Models of non-prioritized belief change: These are models in which the belief change operators considered do not give priority to the new information received (contrary to what is the case regarding the AGM model which fulfils the principle of primacy of new information). For example, the output of a non-prioritized revision may not contain the new belief that has motivated that revision. Analogously, the outcome of a non-prioritized contraction may still contain the sentence by which the contraction is made.

We highlight the following ones from among the operators of the kind mentioned in (a) above:

- Base contraction operators: partial meet base contractions (Hansson, 1992a, 1993, 1991a); kernel contractions and smooth kernel contractions (Hansson, 1994a); and basic AGM-generated base contractions (Fermé, Krevneris, \& Reis, 2008; Fermé, Garapa, \& Reis, 2017).

- Base revision operators: partial meet base revisions (Hansson, 1991a); kernel revisions (Hansson, 1994a; Wassermann, 2000).

1. Throughout this article we will often designate a revision operator simply by a revision. This abuse of language is common in the literature. It will always be clear from the context which meaning is intended for the word revision wherever it shall be used. 
Regarding the kind of operators mentioned in (b), we highlight:

- The pioneering work on non-prioritized contraction where Fermé and Hansson (2001) introduced the concept of shielded contraction. These operators are defined by means of an AGM contraction and a set of sentences, designated by set of retractable sentences and denoted by $R$. If a sentence $\alpha$ belongs to $R$, then the outcome of the shielded contraction by it coincides with the outcome of the associated AGM contraction, otherwise the original belief set is left unchanged.

- The credibility-limited revision operators (CL revision for short), introduced by Hansson et al. (2001). Roughly speaking, a CL revision has the following credibility-based behaviour: If a sentence $\alpha$ is credible, then it is added to the set of beliefs of the agent as a consequence of the revision process, otherwise no change is made to the belief set. Hence, a CL revision operator is induced by a (standard) revision operator and a set of sentences - which contains the sentences that are considered credible, called the set of credible sentences. In the above mentioned paper axiomatic characterizations are presented for CL revision operators induced by AGM revisions and by several kinds of sets of credible sentences. More precisely it presents some results exposing the relation between the postulates satisfied by the CL revision operator and the properties satisfied by the underlying set of credible sentences. CL revision can be seen as a modified version of Makinson's (1997) Screened revision. A Screened revision of a belief set $\mathbf{K}$ by a given sentence $\alpha$ gives rise to a new belief set if the input sentence $\alpha$ is consistent with $A \cap \mathbf{K}$, where $A$ is a set of sentences that are considered immune to revision. If the input sentence is inconsistent with $A \cap \mathbf{K}$ then the belief set $\mathbf{K}$ is left unchanged when the screened revision is performed. The model of CL revision was extended to cover iterated revision by Booth et al. (2012).

At this point we must mention that there are also several papers that combine the two extensions of the AGM model mentioned above. These are works which present operators of non-prioritized belief change for belief bases. We note that the lack of operators of this kind had already been identified by Hansson (1999a) as one of the unexplored regions in the existing works on non-prioritized belief revision.

Concerning non-prioritized contractions for belief bases, Fermé et al. (2003) have presented a class of shielded contractions on belief bases defined by means of a partial meet contraction and a set of retractable sentences (satisfying a certain set of properties). Later, Garapa et al. (2018b) have proposed and axiomatically characterized other (twenty) classes of shielded base contraction induced by several well-known kinds of base contractions (not only partial meet contractions) and several kinds of sets of retractable sentences (i.e. sets satisfying several different, and non-equivalent, sets of properties).

With respect to non-prioritized revisions for belief bases, Fermé et al. (2003) have presented an operator of CL base revision which is induced by a partial meet revision operator and a set of credible sentences satisfying a certain set of properties. ${ }^{2}$ Falappa et al. (2012) axiomatically characterized some operators of non-prioritized multiple revision

2. In that paper only one class of CL base revisions was considered, which is slightly different from all the classes that will be considered in this paper. 
for belief bases. These are operators in which the input is a set of sentences instead of a single sentence and where both the initial belief state and the input set are equally treated.

In the present paper we axiomatically characterize two constructive methods of base revision, namely smooth kernel revisions and basic AGM-generated base revisions. Moreover we will define and thoroughly study twenty classes of CL base revision operators. This paper subsumes and significantly extends the study presented by Garapa et al. (2018a) ${ }^{3}$ and can be seen as the revision counterpart of a paper on shielded base contraction written by the same authors (Garapa et al., 2018b). We will consider classes of CL revisions operators induced by several kinds of base revisions, namely, partial meet base revisions, kernel and smooth kernel revisions and basic AGM-generated base revisions; and several kinds of sets of credible sentences, i.e., we consider several different, and non-equivalent, sets of properties for characterizing a set of credible sentences. We axiomatically characterize all the classes of CL base revisions considered and study the interrelations among them, namely by investigating if each of those classes is or is not (strictly) contained in each one of the remaining classes considered.

The idea of credibility-limit is closely related to that of trust. However, these are two different concepts. In the works that address the notion of trust, credibility is related to the source of the information and not to the information itself. There are several proposals in the belief change literature (e.g., Booth \& Hunter, 2018; Dragoni, Giorgini, \& Baffetti, 1997; Liberatore, 2018; Tamargo, García, Falappa, \& Simari, 2014) which consider the source and the domain of expertise. On the other hand, in the operators considered in this paper the credibility of the new information is "assessed" independently of its source. For example, if a Spanish person asks what the average temperature was during the past summer in Madrid and receives as answer $-32^{\circ} \mathrm{C}$ she will reject this information, independently of its source.

There are several contexts in which CL models can be implemented: In databases, where the integrity constraints cannot be revised during an update, in project management when some restrictions (budget, time) cannot be changed when a contingency plan is designed; as well as all kind of information systems where some constraints cannot be updated or modified.

Example 1.1 When working with databases, it is imperative to detect inconsistent and nonadmissible data and prevent it from being incorporated into the system database. To this end, suitable integrity constraints must be defined. These are used by the system's data validation mechanisms in order to prevent incorrect data from being entered. Integrity constraints are logical conditions that must be satisfied by the input data to be incorporated into the system database. When new input data is received, the database verifies if the integrity constraints are satisfied. If not, the input information is not accepted. For example, a database of people, which includes their birth and death dates (in case that person has already died), should have a rule that for anyone with a death date, that date must be later then their date of birth. If the data entered does not satisfy this rule, then it will not be accepted.

The rest of the paper is organized as follows:

3. In that paper only four of these twenty classes have been considered. Furthermore, only proof sketches were presented for those results. In Appendix A. of this paper we present full proofs for them. 
In Section 2 we introduce the notations and recall the main background concepts and results that will be needed throughout this article. We also recall the main results concerning CL revision operators on belief sets. In Section 3 we introduce and characterize two constructive methods for belief base revision, whose definitions are inspired in two base contraction constructive methods for belief bases. In Section 4 we present a formal definition of credibility-limited base revision and introduce some new belief base revision postulates and some desirable properties that a set of credible sentences should satisfy. Furthermore, we present several results highlighting the interrelations among the properties satisfied by a credibility-limited base revision operator and the properties satisfied by the (standard) revision and the set of credible sentences which induce that credibility-limited revision. Afterwards, in Section 5 we present axiomatic characterizations for the following classes of credibility-limited base revisions: CL revisions induced by partial meet revisions, CL revisions induced by kernel revisions, CL revisions induced by smooth kernel revisions, and CL revisions induced by basic AGM-generated base revisions. For each one of these four classes, we shall identify five different subclasses - each one associated to a certain list of properties of the set of credible sentences. Then, in Section 6 we analyse the interrelations among (all) the classes of CL revisions considered in terms of the relation of (strict) inclusion. Finally, in Section 7 we summarize the main contributions of the paper and briefly discuss their relevance. In Appendix A. we provide proofs for all the original results presented.

\section{Background}

The following subsection introduces the notation that shall be used throughout the article.

\subsection{Formal Preliminaries}

We will assume a propositional language $\mathcal{L}$ that contains the usual truth functional connectives: $\neg$ (negation),$\wedge$ (conjunction),$\vee($ disjunction),$\rightarrow$ (implication) and $\leftrightarrow$ (equivalence). We shall make use of a consequence operation $C n$ that takes sets of sentences to sets of sentences and which satisfies the standard Tarskian properties, namely inclusion, monotony and iteration. Furthermore we will assume that $C n$ satisfies supraclassicality, compactness and deduction. We will sometimes use $C n(\alpha)$ for $C n(\{\alpha\}), A \vdash \alpha$ for $\alpha \in C n(A), \vdash \alpha$ for $\alpha \in C n(\emptyset), A \nvdash \alpha$ for $\alpha \notin C n(A), \forall \alpha$ for $\alpha \notin C n(\emptyset)$. The letters $\alpha, \beta, \ldots$ (except for $\gamma$ and $\sigma$ ) will be used to denote sentences of $\mathcal{L}$. Lowercase Latin letters such as $p, q, \ldots$ will be used to denote atomic sentences of $\mathcal{L}$. $A, B, \ldots$ shall denote sets of sentences of $\mathcal{L}$. $\mathbf{K}$ is reserved to represent a set of sentences that is closed under logical consequence (i.e. $\mathbf{K}=C n(\mathbf{K}))$ - such a set is called a belief set or theory. Given a belief set $\mathbf{K}$ we will use $\mathbf{K}+\alpha$ to denote $C n(\mathbf{K} \cup\{\alpha\})$. We will use the symbols $\star, *, \odot$, and $\circledast$ to denote AGM belief set revision, belief base revision, CL belief set revision and CL belief base revision operators, respectively.

\subsection{AGM Revision Operators}

The operation of revision of a belief set consists of the incorporation of new beliefs in that set. In a revision process, some previous beliefs may be retracted in order to preserve the consistency of the resulting belief set. The following six postulates, which were originally 
presented by Gärdenfors (1988), are commonly known as basic AGM postulates for revision: ${ }^{4}$

$(\star 1) \mathbf{K} \star \alpha=C n(\mathbf{K} \star \alpha)$ (i.e. $\mathbf{K} \star \alpha$ is a belief set).

(Closure)

$(\star 2) \quad \alpha \in \mathbf{K} \star \alpha$.

(Success)

$(\star 3) \mathbf{K} \star \alpha \subseteq \mathbf{K}+\alpha$.

( $\star 4)$ If $\neg \alpha \notin \mathbf{K}$, then $\mathbf{K}+\alpha \subseteq \mathbf{K} \star \alpha$.

(Vacuity)

$(\star 5)$ If $\alpha$ is consistent, then $\mathbf{K} \star \alpha$ is consistent.

(Consistency)

$(\star 6)$ If $\vdash \alpha \leftrightarrow \beta$, then $\mathbf{K} \star \alpha=\mathbf{K} \star \beta$.

(Extensionality)

Definition 2.1 An operator $\star$ for a belief set $\mathbf{K}$ is a basic AGM revision if and only if it satisfies postulates $(\star 1)$ to $(\star 6)$.

Definition 2.2 An operator $\star$ for a belief set $\mathbf{K}$ is an AGM revision if and only if it satisfies postulates $(\star 1)$ to $(\star 6)$ and

$(\star 7) \mathbf{K} \star(\alpha \wedge \beta) \subseteq(\mathbf{K} \star \alpha)+\beta$.

(Superexpansion)

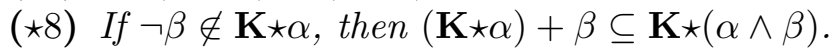

(Subexpansion)

\subsection{Base Revision Operators}

We start this subsection by introducing a definition of a revision operator in terms of postulates. The following definition establishes the minimal set of postulates that a revision operator must satisfy.

Definition 2.3 An operator $*$ for a set $A$ is an operator of revision if and only if * satisfies the following postulates:

(Success) $\alpha \in A * \alpha$.

(Inclusion) $A * \alpha \subseteq A \cup\{\alpha\}$.

(Consistency) If $\alpha \nvdash \perp$, then $A * \alpha \nvdash \perp$.

We now recall several well-known constructive models of revision functions on belief bases.

\subsubsection{PARTial MEeT REVISIONS}

We recall the definition and axiomatic characterization of partial meet revisions.

Definition 2.4 (Alchourrón \& Makinson, 1981) Let $A$ be a belief base and $\alpha$ a sentence. The set $A \perp \alpha$ (A remainder $\alpha)$ is the set of sets such that $B \in A \perp \alpha$ if and only if:

1. $B \subseteq A$.

2. $B \nvdash \alpha$.

4. These postulates had already been presented by Gärdenfors (1982) and by Alchourrón et al. (1985) but with slightly different formulations. 
3. There is no set $B^{\prime}$ such that $B \subset B^{\prime} \subseteq A$ and $B^{\prime} \not \forall \alpha$.

Definition 2.5 (Alchourrón et al., 1985) Let $A$ be a belief base. A selection function for $A$ is a function $\gamma$ such that for all sentences $\alpha$ :

1. If $A \perp \alpha$ is non-empty, then $\gamma(A \perp \alpha)$ is a non-empty subset of $A \perp \alpha$.

2. If $A \perp \alpha$ is empty, then $\gamma(A \perp \alpha)=\{A\}$.

Definition 2.6 (Alchourrón et al., 1985) Let $A$ be a belief base. The partial meet revision operator on $A$ based on a selection function $\gamma$ is the operator $*_{\gamma}$ such that for all sentences $\alpha$ :

$$
A *_{\gamma} \alpha=(\bigcap \gamma(A \perp \neg \alpha)) \cup\{\alpha\} .
$$

An operator $*$ on $A$ is a partial meet revision if and only if there is a selection function $\gamma$ for $A$ such that for all sentences $\alpha: A * \alpha=A *_{\gamma} \alpha$.

In the following observation we recall an axiomatic characterization for partial meet base revision functions.

Observation 2.7 (Hansson, 1991a) Let $A$ be a belief base. An operator $*$ on $A$ is a partial meet revision if and only if $*$ satisfies success, consistency, inclusion and

(Uniformity) If for all subsets $A^{\prime} \subseteq A, A^{\prime} \cup\{\alpha\} \vdash \perp$ if and only if $A^{\prime} \cup\{\beta\} \vdash \perp$, then $A \cap(A * \alpha)=A \cap(A * \beta)$.

(Relevance) If $\beta \in A$ and $\beta \notin A * \alpha$, then there is some $A^{\prime}$ such that $A * \alpha \subseteq A^{\prime} \subseteq A \cup\{\alpha\}, A^{\prime} \forall \perp$ but $A^{\prime} \cup\{\beta\} \vdash \perp$.

Uniformity (Hansson, 1993) states that if two sentences are inconsistent with the same subsets of $A$, then the outcomes of the revisions of $A$ by each of them should keep the same elements of $A$. Relevance (Hansson, 1993) ensures that when revising a set $A$ by a sentence, nothing is removed unless that removal contributes to keeping the outcome of the revision consistent.

\subsubsection{KERNEL REVISIONS}

Hansson (1994a) introduced Kernel Contraction, a generalization of Safe Contraction (Alchourrón \& Makinson, 1985). It is based on a selection among the sentences of a set $A$ that contribute effectively to imply $\alpha$; and on how to use this selection in contracting by $\alpha$. Formally:

Definition 2.8 (Hansson, 1994a) Let $A$ be a set in $\mathcal{L}$ and $\alpha$ a sentence. Then $A \Perp \alpha$ is the set such that $B \in A \Perp \alpha$ if and only if:

1. $B \subseteq A$.

2. $B \vdash \alpha$.

3. If $B^{\prime} \subset B$ then $B^{\prime} \nvdash \alpha$. 
$A \Perp \alpha$ is called the kernel set of $A$ with respect to $\alpha$ and its elements are the $\alpha$-kernels of $A$.

To contract a belief $\alpha$ from a set $A$ one must give up sentences in each $\alpha$-kernel, otherwise $\alpha$ would continue being implied by $A$. The so-called incision functions select the beliefs to be discarded.

Definition 2.9 (Hansson, 1994a) Let $A$ be a set of sentences. Let $A \Perp \alpha$ be the kernel set of $A$ with respect to $\alpha$. An incision function $\sigma$ for $A$ is a function such that for all sentences $\alpha$ :

1. $\sigma(A \Perp \alpha) \subseteq \bigcup(A \Perp \alpha)$.

2. If $\emptyset \neq B \in A \Perp \alpha$, then $B \cap \sigma(A \Perp \alpha) \neq \emptyset$.

Definition 2.10 Let $A$ be a belief base. The kernel revision operator on $A$ based on an incision function $\sigma$ is the operator $*_{\sigma}$ such that for all sentences $\alpha$ :

$$
A *_{\sigma} \alpha=(A \backslash \sigma(A \Perp \neg \alpha)) \cup\{\alpha\} .
$$

Observation 2.11 (Wassermann, 2000) Let $A$ be a belief base. An operator $*$ on $A$ is a kernel revision function for $A$ if and only if $*$ satisfies success, consistency, inclusion, uniformity and

(Core-retainment) If $\beta \in A$ and $\beta \notin A * \alpha$, then there is some $A^{\prime} \subseteq A$ such that $A^{\prime} \forall \neg \alpha$ and $A^{\prime} \cup\{\beta\} \vdash \neg \alpha .^{5}$

Core-retainment (Wassermann, 2000) is, as relevance, an expression of the principle of minimal change. Core-retainment follows from relevance and success.

\subsection{Credibility-Limited Revision for Belief Sets}

Credibility-limited revision (Hansson et al., 2001) is an operator of non-prioritized revision. When revising a belief set by a sentence, we need to analyse if this sentence is credible or not. When revising by a credible sentence, the operator works as a basic AGM revision operator, otherwise it leaves the original belief set unchanged. Formally:

Definition 2.12 (Hansson et al., 2001) Let $\mathbf{K}$ be a belief set, $\star$ a basic AGM revision operator on $\mathbf{K}$ and $C$ a subset of $\mathcal{L}$ (the set of credible sentences). Then $\odot$ is a credibilitylimited revision operator induced by $\star$ and $C$ if and only if:

$$
\mathbf{K} \odot \alpha= \begin{cases}\mathbf{K} \star \alpha & \text { if } \alpha \in C \\ \mathbf{K} & \text { otherwise }\end{cases}
$$

5. To be more precise we note that this axiomatic characterization is equivalent to the one actually presented by Wassermann (2000), which uses the postulate of non-contradiction (if $\forall \neg \alpha$, then $A * \alpha \forall \neg \alpha$ ) instead of consistency. 
This construction can be further specified by adding constraints to the structure of $C$ (the set of credible sentences). ${ }^{6}$ Hansson et al. (2001) proposed the following desirable properties for $C$ :

Credibility of Logical Equivalents: If $\vdash \alpha \leftrightarrow \beta$, and $\alpha \in C$, then $\beta \in C$.

Single Sentence Closure: If $\alpha \in C$, then $C n(\alpha) \subseteq C$.

Disjunctive Completeness: If $\alpha \vee \beta \in C$, then either $\alpha \in C$ or $\beta \in C$.

Negation Completeness: $\alpha \in C$ or $\neg \alpha \in C$.

Element Consistency: If $\alpha \in C$, then $\alpha \nvdash \perp$.

Expansive Credibility: If $\mathbf{K} \nvdash \alpha$, then $\neg \alpha \in C$.

Revision Credibility: If $\alpha \in C$, then $\mathbf{K} \odot \alpha \subseteq C$.

Credibility of logical equivalents also designated by closure under logical equivalence, states that logically equivalent sentences should be both elements of $C$ or of $\mathcal{L} \backslash C$. Single sentence closure says that if a sentence is credible then all its logical consequences are also credible. Single sentence closure implies closure under logical equivalence. Disjunctive completeness states that if two sentences are not credible, then their disjunction is not credible. Negation completeness states that for any sentence it holds that either it is credible or its negation is credible. Element consistency states that contradictions are not credible. Expansive credibility informally states that sentences that are consistent with $\mathbf{K}$ are credible. Revision credibility states that sentences in the outcome of a revision by a credible sentence are credible.

When considering a credibility-limited revision the success postulate must be discarded. It must be replaced by weaker properties. The following postulates were formulated by Hansson et al. (2001) and by Fermé and Hansson (2001):

(Relative Success) $\alpha \in \mathbf{K} \odot \alpha$ or $\mathbf{K} \odot \alpha=\mathbf{K}$.

(Disjunctive Success) Either $\alpha \in \mathbf{K} \odot \alpha$ or $\neg \alpha \in \mathbf{K} \odot \neg \alpha$.

(Strict Improvement) If $\alpha \in \mathbf{K} \odot \alpha$ and $\vdash \alpha \rightarrow \beta$, then $\beta \in \mathbf{K} \odot \beta$.

(Regularity) If $\beta \in \mathbf{K} \odot \alpha$, then $\beta \in \mathbf{K} \odot \beta$.

(Strong Regularity) If $\neg \beta \notin \mathbf{K} \odot \alpha$, then $\beta \in \mathbf{K} \odot \beta$.

(Disjunctive Distribution) If $\alpha \vee \beta \in \mathbf{K} \odot(\alpha \vee \beta)$, then $\alpha \in \mathbf{K} \odot \alpha$ or $\beta \in \mathbf{K} \odot \beta$. (Disjunctive Constancy) If $\mathbf{K} \odot \alpha=\mathbf{K} \odot \beta=\mathbf{K}$, then $\mathbf{K} \odot(\alpha \vee \beta)=\mathbf{K}$.

Relative success states that either a sentence is incorporated in the revision of a belief set by it, or the original belief set is left unchanged. Disjunctive success states that either a sentence belong to the revision of a belief set by it or the negation of that sentence belongs to the revision of that belief set by it. Strict improvement states that if a certain sentence is incorporated when revising a belief set by it, then the same thing happens regarding every logical consequence of that sentence. Regularity says that if a sentence does not belong to the revision of a belief set by it, then that sentence does not belong to the revision of that belief set by any other sentence. Strong regularity states that if a sentence does not belong to the revision of a belief set by it, then its negation belongs to the revision of that belief

6. One of the assumptions of this paper is that the set $C$ is given a priori. 
set by any given sentence. Disjunctive distribution states that if a disjunction belongs to the revision of a belief set by it, then the same thing happens regarding at least one of its disjuncts. Disjunctive constancy is, as disjunctive distribution, a postulate concerning revision by disjunctions. It states that a belief set is left unchanged when revising it by a disjunction, whenever the same thing occurs when revising that belief set by either one of the two disjuncts.

The following postulates are related to consistency. They will also be used in the axiomatic characterizations that we will present.

(Weak Consistency Preservation) (Katsuno \& Mendelzon, 1992) If both K and $\alpha$ are consistent, then so is $\mathbf{K} \odot \alpha$.

(Strong Consistency) (Hansson, 1996) $\mathbf{K} \odot \alpha$ is consistent.

(Consistency Preservation) (Makinson, 1997) If $\mathbf{K}$ is consistent, then $\mathbf{K} \odot \alpha$ is consistent.

(Consistent Expansion) (Fermé \& Hansson, 1999) If $\mathbf{K} \nsubseteq \mathbb{K} \odot \alpha$, then $\mathbf{K} \cup(\mathbf{K} \odot \alpha) \vdash \perp$.

Additionally, Hansson et al. (2001) have proposed the following postulate, that consists of an adaptation of subexpansion to the context of non-prioritized revision:

(Guarded Subexpansion) If $\alpha \in \mathbf{K} \odot \alpha$ and $\mathbf{K} \odot \alpha \not \neg \neg \beta$, then $(\mathbf{K} \odot \alpha)+\beta \subseteq \mathbf{K} \odot(\alpha \wedge \beta)$.

Guarded subexpansion and subexpansion are equivalent in the presence of success.

Now we are in conditions to recall the representations theorems for the credibility-limited revision operators mentioned above. We will start by presenting a minimal representation theorem. After that, by adding conditions on $C$, the set of credible sentences, we obtain more specific representation theorems.

Observation 2.13 (Hansson et al., 2001) Let $\mathbf{K}$ be a consistent belief set and $\odot$ an operator on $\mathbf{K}$. Then the following three conditions are equivalent:

1. $\odot$ satisfies closure, relative success, inclusion, weak consistency preservation, consistent expansion and extensionality.

2. $\odot$ is an operator of credibility-limited revision induced by a basic AGM revision operator for $\mathbf{K}$ and a set $C \subseteq \mathcal{L}$ that is closed under logical equivalence.

3. $\odot$ is an operator of credibility-limited revision induced by a basic AGM revision operator for $\mathbf{K}$ and a set $C \subseteq \mathcal{L}$ that satisfies $\mathbf{K} \subseteq C$ and is closed under logical equivalence.

Observation 2.14 (Hansson et al., 2001) Let $\mathbf{K}$ be a consistent belief set and $\odot$ an operator on $\mathbf{K}$. Then: ${ }^{7}$

7. The schema presented in this observation (and whenever a similar schema is used) should be interpreted as follows: 


\begin{tabular}{|c|c|}
\hline $\begin{array}{c}\odot \text { is an operator of credibility-limited } \\
\text { revision induced by a basic AGM } \\
\text { operator on } \mathrm{K} \text { and } \text { a set } C \subseteq \mathcal{L} \text { that } \text { is } \\
\text { closed under logical equivalence and } \\
\text { satisfies }\end{array}$ & $\begin{array}{c}\text { if and only if } \odot \text { satisfies closure, } \\
\text { relative } \text { success, inclusion, weak } \\
\text { consistency } \text { preservation, } \text { consistent } \\
\text { expansion, } \text { extensionality } \text { and }\end{array}$ \\
\hline single sentence closure & strict improvement \\
\hline disjunctive completeness & disjunctive distribution \\
\hline negation completeness & disjunctive success \\
\hline element consistency & strong consistency \\
\hline expansive credibility & vacuity \\
\hline
\end{tabular}

\section{Two New Constructive Methods for Belief Base Revision}

In this section we present two new constructive methods for belief base revision. Both methods are the revision counterpart of existing constructive methods for belief base contraction. After that we will analyze the different belief base revision operators from the point view of the notion of minimal change.

\subsection{Smooth Kernel Revisions}

The following definition introduces the concept of smooth kernel base revision which is a kernel base revision based on a smooth incision function.

Definition 3.1 (Hansson, 1994a) An incision function $\sigma$ for a set $A$ is smooth if and only if it holds for all subsets $A^{\prime}$ of $A$ that if $A^{\prime} \vdash \beta$ and $\beta \in \sigma(A \Perp \alpha)$ then $A^{\prime} \cap \sigma(A \Perp \alpha) \neq \emptyset$. $A$ kernel revision is smooth if and only if it is based on a smooth incision function.

In the following theorem an axiomatic characterization is provided for smooth kernel base revisions. $^{8}$

Theorem 3.2 Let $A$ be a belief base. An operator $*$ on $A$ is a smooth kernel revision if and only if it satisfies success, consistency, inclusion, uniformity, core-retainment and (Weak Relative Closure) $A \cap C n(A \cap A * \alpha) \subseteq A * \alpha$.

- $\odot$ is an operator of credibility-limited revision induced by a basic AGM operator on $\mathbf{K}$ and a set $C \subseteq \mathcal{L}$ that is closed under logical equivalence and satisfies single sentence closure iff $\odot$ satisfies closure, relative success, inclusion, weak consistency preservation, consistent expansion, extensionality and strict improvement;

- $\odot$ is an operator of credibility-limited revision induced by a basic AGM operator on $\mathbf{K}$ and a set $C \subseteq \mathcal{L}$ that is closed under logical equivalence and satisfies disjunctive completeness iff $\odot$ satisfies closure, relative success, inclusion, weak consistency preservation, consistent expansion, extensionality and disjunctive distribution;

$-\ldots$

8. This result was presented without a full proof by Garapa et al. (2018b). In the Appendix of this paper we present a full proof for this result. 
Weak relative closure, which is an adaptation, for revision, of the contraction postulate of relative closure (Hansson, 1994a), states that the set formed by the elements of $A$ that are included in the outcome of revising $A$ by $\alpha$ is logically closed relative to $A .{ }^{9}$ We note that the intersection with the set $A$ that appears in the argument of consequence operator $C n$ is not irrelevant as one might think. To see this consider the following example: Let $A=\{\alpha \rightarrow \beta, \beta, \beta \rightarrow \neg \alpha\}$ and $A * \alpha=\{\alpha \rightarrow \beta, \alpha\}$. Hence $\beta \in A \cap C n(A * \alpha)$ but $\beta \notin A * \alpha$. On the other hand, $\alpha \rightarrow \beta$ is the only element of $A$ that can be deduced from $A \cap A * \alpha$. It holds that $\alpha \rightarrow \beta \in A * \alpha$. Thus, $*$ satisfies weak relative closure but not the property $A \cap C n(A * \alpha) \subseteq A * \alpha$.

\subsection{Basic AGM-Generated Base Revisions}

We will now recall the definition and an axiomatic characterization for basic AGM-generated base revisions, which are operators of base revision defined from operators of basic AGM revision (for belief sets).

Definition 3.3 Let $A$ be a belief base. An operator $*$ for $A$ is a basic AGM-generated base revision if and only if there exists some basic $A G M$ revision $\star$ for $C n(A)$, such that for all $\alpha \in \mathcal{L}:$

$$
A * \alpha=(C n(A) \star \alpha) \cap(A \cup\{\alpha\}) .
$$

This function is the revision counterpart of AGM-generated base contraction presented by Ferm'e et al. (2008). In the following Theorem an axiomatic characterization is provided for smooth kernel base revisions. ${ }^{10}$

Theorem 3.4 Let $A$ be a belief base. An operator * on $A$ is a basic AGM-generated base revision if and only if it satisfies success, consistency, inclusion and

(Vacuity) If $A \nvdash \neg \alpha$, then $A \cup\{\alpha\} \subseteq A * \alpha$.

(Weak Extensionality) If $\vdash \alpha \leftrightarrow \beta$, then $A \cap A * \alpha=A \cap A * \beta$.

(Disjunctive Elimination) If $\beta \in A$ and $\beta \notin A * \alpha$, then $A * \alpha \forall \neg \alpha \vee \beta$.

The formulation of vacuity consists in an adaptation to belief bases of postulate $(\star 4)$. It states that if a sentence $\alpha$ is consistent with $A$ that the outcome of contracting $A$ by $\alpha$ contains $A \cup\{\alpha\}$. If both vacuity and inclusion are satisfied then such outcome coincides precisely with $A \cup\{\alpha\}$.

Weak extensionality states that if $\alpha$ and $\beta$ are two logically equivalent beliefs then every element of $A$ that is kept when revising by $\alpha$ is also kept when revising by $\beta$. We note that weak extensionality is a weaker version of extensionality: If $\vdash \alpha \leftrightarrow \beta$, then $A * \alpha=A * \beta$. We also note that, in general, extensionality is not satisfied by belief base revisions. The following example illustrates this fact: Let $\alpha$ and $\beta$ be two distinct sentences such that $\vdash \alpha \leftrightarrow \beta$. Let $A$ be a belief base such that $A \cap\{\alpha, \beta\}=\emptyset$. Let $*$ be a revision operator on $A$, thus $*$ satisfies success and inclusion. Then $\alpha \in A * \alpha$ but $\alpha \notin A * \beta$, therefore $A * \alpha \neq A * \beta$.

9. A set $A$ is logically closed relative to $B$ if and only if $C n(A) \cap B \subseteq A$ (Hansson, 1991b).

10. The remark concerning Theorem 3.2 written in Footnote 8 also applies to this result. 
Disjunctive elimination is an adaptation, for revision, of the contraction postulate with the same designation proposed by Fermé et al. (2008) and states that if $\beta$ is removed when revising a set $A$ by $\alpha$, then from the revision of $A$ by $\alpha$ we can not deduce that $\alpha$ implies $\beta$.

\subsection{Belief Bases and Minimal Change}

One of the hallmarks of the AGM model is that partial meet revision, kernel revision, smooth kernel revision and basic AGM-generated base revisions are all equivalent when applied to belief sets. ${ }^{11}$ However, partial meet revision and kernel revision are based in two different notions of minimal change; the first one in a selection of remainder sets, the latter on an incision function on kernel sets. In what concerns basic AGM-generated base revisions, it inherits the minimal change of the AGM function on which it is based.

This diversity of models is present in the literature and there is not a general consensus about which one better addresses the principle of minimal change. ${ }^{12}$ In this subsection we present the existing relations between the different constructive models when applied to belief bases.

The following observation exposes some relations among some of the postulates used in the axiomatic characterizations of the different classes of base revision that were mentioned above. $^{13}$

Observation 3.5 Let $A$ be a belief base and $*$ be an operator on $A$.

(a) If $*$ satisfies uniformity, then $*$ satisfies weak extensionality.

(b) If * satisfies disjunctive elimination, then $*$ satisfies weak relative closure.

(c) If * satisfies relevance and success, then * satisfies core-retainment and disjunctive elimination.

(d) If * satisfies success and core-retainment, then $*$ satisfies vacuity.

The following observation exposes the interrelations among the different classes of revisions mentioned above which follow trivially from their axiomatic characterizations presented in Observations 2.7 and 2.11, and in Theorems 3.2 and 3.4 and the interrelations among postulates presented in Observation 3.5.

Observation 3.6 Let $A$ be a belief base and * be a revision operator on A. Then:

(a) If $*$ is an operator of partial meet revision, then it is an operator of smooth kernel revision.

11. The proof of this claim is relatively simple. Hansson (1994a) proved that the class of kernel contraction and smooth kernel contraction are equivalent to the class of partial meet contraction for belief sets. Fermé et al. (2008) proved that the class of basic AGM-generated base contraction is equivalent with the class of partial meet contraction. Finally, the four corresponding models of belief set revision are defined from the contraction operators by means of the Levi identity $(\mathbf{K} \star \alpha=\mathbf{K}-\neg \alpha+\alpha)$.

12. For this reason we consider all those four classes of base revision operators in this paper.

13. Some of the relations among postulates stated in the following observation have been presented without a proof by Garapa et al. (2018a). In this paper we present a full proof for this result. 
(b) If * is an operator of smooth kernel revision, then it is an operator of kernel revision.

(c) If $*$ is an operator of partial meet revision, then it is an operator of basic AGM-generated base revision.

Figure 1 illustrates the relations exposed in the observation above.

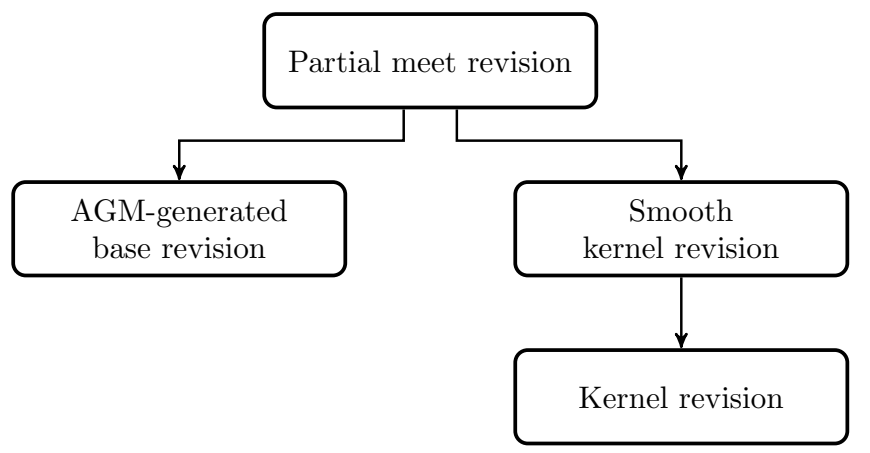

Figure 1: Map among different classes of base revision operators.

\section{Credibility-Limited Base Revisions}

In this section we present the basic ideas underlying the construction of credibility-limited base revisions. Credibility-limited base revision can be seen as functions defined in two steps. In the first step, one needs to determine which sentences are credible, i.e., the sentences that an agent is willing to incorporate when performing a revision. Afterwards the function should:

- leave the set of beliefs unchanged when revising it by a non-credible sentence;

- work as a base revision when revising by a credible sentence.

The following definition formalizes this concept:

Definition 4.1 Let $*$ be a revision operator (i.e., an operator that satisfies success, inclusion and consistency) on a belief base A. Let $C$ be a set of sentences (the associated set of credible sentences). Then $\circledast$ is the credibility-limited base revision induced by $*$ and $C$ if and only if:

$$
A \circledast \alpha= \begin{cases}A * \alpha & \text { if } \alpha \in C \\ A & \text { otherwise }\end{cases}
$$

The above definition is an extension of the one presented for credibility-limited base revision by Fermé et al. (2003), since it defines a credibility-limited base revision induced by a generic revision operator (and a set $C$ ) instead of by a partial meet revision. The following example illustrates the behaviour of a credibility-limited base revision. 
Example 4.2 (Garapa et al., 2018b) Let $A=\{p, p \vee \neg q, p \rightarrow \neg q\}$, Cn be purely truthfunctional and $*$ be a partial meet base revision on $A$. It holds that $A \perp \neg q=\{\{p, p \vee$ $\neg q\},\{p \rightarrow \neg q\}\}$. Let $\gamma$ be a selection function for $A$ such that $\gamma(A \perp \neg q)=\{\{p, p \vee \neg q\}\}$. Hence $A * q=\{p, p \vee \neg q, q\}$. Let $C=C n(q) \cup C n(p) \cup C n(\neg q)$ and $\circledast$ be the credibility-limited base revision induced by $*$ and $C$. It holds that $p \wedge q \notin C$, hence $A \circledast(p \wedge q)=A$. On the other hand $q \in C$. Hence $A \circledast q=A * q=\{p, p \vee \neg q, q\}$.

\subsection{Two Dimensions for Analysing Credibility-Limited Base Revisions}

Definition 4.1 claims that $\circledast$ is induced by $*$ and $C$. For this reason the properties of the $\circledast$ revision operator are determined by two different factors:

1. The properties satisfied by the set $C$;

2. The properties of the belief base revision $*$.

In subsection 3.3 we discussed that in belief bases, contrary to what is the case in the context of belief sets, different methods encompass different notions of minimal change. This means that using different belief revision functions $*$, combined with different properties of $C$ will lead to a different set of properties satisfied by the $\circledast$ operator. Since the study of base revision operators is an active research topic, it is convenient to study what is the impact, in terms of the properties satisfied by $\circledast$, of each single postulate satisfied by $*$ (rather than only studying which properties as a whole are satisfied by $\circledast$ when $*$ is one of the four kinds of base revision which we have mentioned above).

The operator $\circledast$ differs from the AGM functions in two fundamental issues: It may not satisfy the postulate of success, i.e., the new information may not be accepted; and it fulfils a stronger consistency condition/requirement, namely that the outcome of a change must be a consistent belief base, whatever is the input sentence that causes that revision. On the other hand, it is also important to study the relation between the (properties of the) set $C$ and the base revision $*$ which (together) induce the operator $\circledast$.

In the remainder of this section we will focus in which properties are suitable for the set $C$ in the context of belief bases and how these properties, combined with properties of $*$ influence the properties of the induced operator $\circledast$.

\subsection{Credible Sentences}

In this section we will analyse plausible properties for the set $C$ in the belief base revision framework. We start by pointing out that the full logical closure, i.e., $\operatorname{Cn}(C) \subseteq C$ is patently unreasonable; two sentences $\alpha$ and $\beta$ may be credible without $\alpha \wedge \beta$ being so (an obvious example is to consider $\beta$ such that $\vdash \beta \leftrightarrow \neg \alpha$ ). In what follows, we present different properties that relax this condition.

The minimal requirement is that $C$ must be closed under double negation:

Closure Under Double Negation: $\alpha \in C$ if and only if $\neg \neg \alpha \in C$. 
We can extend this condition to require that, regarding credibility, equivalent sentences have the same status:

Credibility of Logical Equivalents (Hansson et al., 2001): If $\vdash \alpha \leftrightarrow \beta$, and $\alpha \in C$, then $\beta \in C$.

If we assign the status of "credible" to a sentence $\alpha$ it is reasonable to expect that its consequences are also credible:

Single Sentence Closure (Hansson et al., 2001): If $\alpha \in C$, then $C n(\alpha) \subseteq C$.

Note that if a set $C$ satisfies single sentence closure then it also satisfies credibility of logical equivalents. If two sentences are not credible, then their disjunction is not credible:

Disjunctive Completeness (Hansson et al., 2001): If $\alpha \vee \beta \in C$, then either $\alpha \in C$ or $\beta \in C$.

An immediate consequence of the two previous conditions, when $C \neq \emptyset$, is the following:

Negation Completeness (Hansson et al., 2001): $\alpha \in C$ or $\neg \alpha \in C$.

Regarding consistency, it is expected that contradictions are not credible:

Element Consistency (Hansson et al., 2001): If $\alpha \in C$, then $\alpha \nvdash \perp$.

The previous properties are internal in the sense that they only present relations among elements of the set $C$. The following properties relate the set $C$ with the set $A .{ }^{14}$ If a sentence is consistent with $A$, then this sentence must be credible:

Expansive Credibility (Hansson et al., 2001): If $A \nvdash \alpha$, then $\neg \alpha \in C$.

Another reasonable property is that all the logical consequences of $A$ are credible when $A$ is consistent:

Credibility Lower Bounding: If $A$ is consistent, then $C n(A) \subseteq C$.

If two sentences have the "same behaviour" regarding a set $A$, i.e., are consistent with exactly the same subsets of $A$, it is expected that both have the same status regarding $C$ :

Uniform Credibility: If it holds for all subsets $A^{\prime}$ of $A$ that $A^{\prime} \cup\{\alpha\} \vdash \perp$ if and only if $A^{\prime} \cup\{\beta\} \vdash \perp$, then $\alpha \in C$ if and only if $\beta \in C$.

14. We note that more rigorously the expression "with respect to $A$ " should be added to the designation of these properties. This will be omitted since there is no risk of ambiguity whenever these properties are mentioned along this paper. 
Finally, the following properties relate the set $C$ with $A$ and the operator $\circledast .{ }^{15} \mathrm{~A}$ reasonable requirement is that all the sentences in the outcome of a revision by a credible sentence are credible.

Revision Credibility (Hansson et al., 2001): If $\alpha \in C$, then $A \circledast \alpha \subseteq C$.

On the other hand, it is expected that all the non-credible sentences are inconsistent with the outcome of any revision::

Strong Revision Credibility (Fermé et al., 2003): If $\alpha \notin C$, then $A \circledast \beta \vdash \neg \alpha$.

A stronger requirement states that if a sentence $\alpha$ is not credible, then any possible revision keeps a subset of $A$ that implies $\neg \alpha$ :

Strong Expansive Credibility: If $\alpha \notin C$, then $A \cap A \circledast \beta \vdash \neg \alpha$.

Finally we introduce a condition that consists of a relation between the set of credible sentences $C$ and a (standard) base revision $*$ on a set $A$ (which may be combined for inducing a CL base revision on $A$ ).

$$
\text { If } \alpha \notin C \text { and } \beta \in C \text {, then } A \cap A * \beta \vdash \neg \alpha \text {. }
$$

Condition $(\mathbf{C}-*)$ states that if a sentence $\alpha$ is not credible, then any possible outcome of revising a set $A$ by a credible sentence contains a subset of $A$ that implies $\neg \alpha$.

The following observation exposes that in the presence of closure under double negation, the property of strong expansive credibility is equivalent to the conjunction of expansive credibility with condition $(\mathbf{C}-*)$.

Observation 4.3 Let $A$ be a belief base and $\circledast$ be a credibility-limited base revision induced by a revision operator $*$ on $A$ and a set $C \subseteq \mathcal{L}$ that satisfies closure under double negation. $C$ satisfies strong expansive credibility if and only if $C$ satisfies expansive credibility and * and $C$ satisfy condition $(\mathbf{C}-*)$.

According to the above result, given a credibility-limited base revision $\circledast$ induced by a revision operator $*$ and a set (of credible) sentences $C$, in the presence of expansive credibility and closure under double negation, the properties of strong expansive credibility and $(\mathbf{C}-*)$ are equivalent. Nevertheless, we note that, condition $(\mathbf{C}-*)$ relates two structures (namely $*$ and $C$ ) that are independent of each other, while, on the other hand, strong expansive credibility presents a relation between a credibility limited revision $\circledast$ and its associated set of credible sentences. Therefore, in the process of constructing a credibility-limited base revision it is more natural to consider condition $(\mathbf{C}-*)$ than strong

15. We note that more rigorously the expression "with respect to $A$ and $\circledast$ " should be added to the designation of these properties. This will be omitted since there is no risk of ambiguity whenever these properties are mentioned along this paper. 
expansive credibility.

The following observations illustrate some relations between properties of sets of credible sentences:

Observation 4.4 Let $C$ be a subset of $\mathcal{L}$ that satisfies disjunctive completeness and element consistency. Then $C$ satisfies negation completeness if and only if $C n(\emptyset) \subseteq C$.

Observation 4.5 Let $C$ be a set of sentences.

(a) If $C$ satisfies single sentence closure, then $C$ satisfies credibility of logical equivalents.

(b) If $C$ satisfies uniform credibility, then $C$ satisfies credibility of logical equivalents.

(c) If $C$ satisfies expansive credibility and credibility lower bounding with respect to a consistent set $A$, then $C$ satisfies negation completeness.

(d) If $\alpha \in C n(\emptyset)$ and $C$ satisfies negation completeness and element consistency, then $\alpha \in C$.

(e) If $C$ satisfies credibility of logical equivalents, then $C$ is closed under double negation.

\subsection{Postulates for Credibility-Limited Base Revisions}

In this subsection we recall some postulates for (credibility-limited) base revision and some relations among these postulates. Most of the following postulates are an adaptation to the belief bases context of the postulates for credibility-limited revisions on belief sets presented in Subsection 2.2.

We start by mentioning that, in the context of credibility-limited base revision, there may be some sentences that will not be accepted. This means that the success postulate $(\alpha \in A \circledast \alpha)$ may not hold. Thus, we must consider weaker versions of the success postulate capable of capturing the intuitions underlying CL revisions. The first one is about the general principle of credibility-limited base revision: a sentence is accepted or no change is made:

(Relative Success) $\alpha \in A \circledast \alpha$ or $A \circledast \alpha=A$.

If we are willing to accept a sentence $\alpha$ it is reasonable to accept all the sentences that are logical consequence of $\alpha$ :

(Strict Improvement) If $\alpha \in A \circledast \alpha$ and $\vdash \alpha \rightarrow \beta$, then $\beta \in A \circledast \beta$.

If a disjunction belongs to the revision of a belief set by it, then the same thing should happen regarding at least one of its disjuncts.

(Disjunctive Distribution) If $\alpha \vee \beta \in A \circledast(\alpha \vee \beta)$, then $\alpha \in A \circledast \alpha$ or $\beta \in A \circledast \beta$. 
The two following postulates specify the behaviour of CL revisions when it is the case that a certain sentence $\beta$ is not acceptable, i.e., $\beta \notin A \circledast \beta$. The first one states that when that is the case, $\beta$ is not accepted as a result of a revision by any other sentence. The second one imposes that, in that case, $\neg \beta$ is implied by the outcome of the revision by any sentence:

(Regularity) If $A \circledast \alpha \vdash \beta$, then $\beta \in A \circledast \beta$.

(Strong Regularity) If $A \circledast \alpha \forall \neg \beta$, then $\beta \in A \circledast \beta$.

The following postulate, originally proposed by Garapa et al. (2018a), states that if the formulae of $A$ that are kept when revising it by a sentence $\beta$ imply $\neg \beta$, then $\neg \beta$ is implied by the formulae of $A$ that remain when revising it by any formula.

(Persistence) If $\neg \beta \in C n(A \cap A \circledast \beta)$, then $\neg \beta \in C n(A \cap A \circledast \alpha)$.

Although persistence is a reasonable property, there are some contexts in which this postulate is not satisfied, as it is the case in the following situation. ${ }^{16}$ Suppose I believe strongly in $\neg \beta$ and that at a certain moment I get the information that $\beta$ is the case. I am not willing to stop believing in $\neg \beta$ in order to incorporate $\beta$. Suppose now that I get stronger information that proves that $\beta$ is the case (for example, $\alpha \wedge(\alpha \rightarrow \beta)$ ). So, although I revise by a belief other than $\beta$, I end up losing my belief in $\neg \beta$. For example, suppose John made a retreat away from the rest of the world between December 2019 and May 2020, and did not have access to any information during that period. Suppose now that when he returns to civilization, in June 2020, we tell him that the streets of some of the main cities of the world have been completely empty for several weeks in March-April 2020. John will not believe it. Then we explain him that this happened because a virus appeared in December 2019 and to avoid contamination, a large part of the population had to be confined at home. Then John may recall hearing in the news, before going to the retreat, that a highly contagious virus had been discovered. At that moment John will start to believe that the streets of some of the main cities in the world have been completely empty for some time during his retreat.

In AGM base revision, the outcome is consistent unless the input is inconsistent itself. This is because in that model the principle of primacy of the new information has priority over the principle of consistency. Since in what concerns credibility-limited base revision the former principle is not valid, it is reasonable to require a stronger consistency condition:

(Consistency Preservation) If $A \nvdash \perp$, then $A \circledast \alpha \nvdash \perp$.

The following observations relate some of the postulates mentioned above.

Observation 4.6 Let $A$ be a belief base and $\circledast$ be an operator on $A$. If $\circledast$ satisfies relevance and relative success, then $\circledast$ satisfies disjunctive elimination and core-retainment.

16. For this reason, we will consider several classes of credibility-limited base revision, some of which satisfy persistence and others that do not. 
Observation 4.7 Let $A$ be a consistent belief base and $\circledast$ an operation on $A$. If $\circledast$ satisfies consistency preservation, persistence, relative success and vacuity, then $\circledast$ satisfies disjunctive distribution, strict improvement and regularity.

\subsection{Relations Between Base Revisions and Credibility-Limited Base Revisions}

The following observation illustrates some properties that an operator of credibility-limited base revision, induced by a revision operator $*$ and a set $C$, satisfies whenever $*$ and $C$ satisfy some given properties.

Observation 4.8 Let $A$ be a belief base, $C \subseteq \mathcal{L}$, and $\circledast$ be a credibility-base revision induced by a revision operator $*$ and $C$. Then:

(a) It holds that:

\begin{tabular}{|c|c|}
\hline If $*$ satisfies & then $\circledast$ satisfies \\
\hline- & inclusion and relative success \\
\hline relevance & relevance \\
\hline core-retainment & core-retainment \\
\hline disjunctive elimination & disjunctive elimination \\
\hline weak relative closure & weak relative closure \\
\hline
\end{tabular}

(b) If $C$ satisfies element consistency, then:

\begin{tabular}{|c|c|}
\hline If $*$ satisfies & then $\circledast$ satisfies \\
\hline- & consistency preservation \\
\hline
\end{tabular}

(c) If $C$ satisfies uniform credibility, then:

\begin{tabular}{|c|c|}
\hline If $*$ satisfies & then $\circledast$ satisfies \\
\hline uniformity & uniformity \\
\hline
\end{tabular}

(d) If C satisfies credibility of logical equivalents (or single sentence closure), then:

\begin{tabular}{|c|c|}
\hline If $*$ satisfies & then $\circledast$ satisfies \\
\hline weak extensionality & weak extensionality \\
\hline
\end{tabular}

(e) If C satisfies expansive credibility and is closed under double negation (or satisfies either credibility of logical equivalents or uniform credibility or single sentence closure), then:

\begin{tabular}{|c|c|}
\hline If $*$ satisfies & then $\circledast$ satisfies \\
\hline vacuity & vacuity \\
\hline
\end{tabular}

(f) Let $A \nvdash \perp$. If $C$ satisfies expansive credibility and single sentence closure, then:

\begin{tabular}{|c|c|}
\hline If $*$ satisfies & then $\circledast$ satisfies \\
\hline- & strict improvement \\
\hline
\end{tabular}

(g) Let $A \nvdash \perp$. If $C$ satisfies expansive credibility, closure under double negation (or credibility of logical equivalents or uniform credibility or single sentence closure) and disjunctive completeness, then: 


\begin{tabular}{|c|c|}
\hline If $*$ satisfies & then $\circledast$ satisfies \\
\hline- & disjunctive distribution \\
\hline
\end{tabular}

(h) If $C$ and $*$ satisfy condition $(\mathbf{C}-*)$ and $C$ satisfies element consistency, then:

\begin{tabular}{|c|c|}
\hline If $*$ satisfies & then $\circledast$ satisfies \\
\hline- & persistence \\
\hline weak extensionality & weak extensionality \\
\hline uniformity & uniformity \\
\hline
\end{tabular}

(i) If $C$ and $*$ satisfy condition $(\mathbf{C}-*)$ and $C$ satisfies expansive credibility, then:

\begin{tabular}{|c|c|}
\hline If $*$ satisfies & then $\circledast$ satisfies \\
\hline vacuity & vacuity \\
\hline
\end{tabular}

If $\alpha$ is credible, then it should be an element of the outcome of the revision of a set $A$ by it. Therefore, a natural way to define a set of credible sentences $C$ is by $C=\{\alpha: \alpha \in A \circledast \alpha\}$, where $\circledast$ is a credibility-limited revision. The following theorem states that, if the set $C$ satisfies expansive credibility and closure under double negation, then $C$ is precisely the set that is explicitly defined in terms of $\circledast$ by the above identity.

Theorem 4.9 Let $A$ be a consistent belief base and $\circledast$ be an operator of credibility-limited revision induced by a revision operator for $A$ and a set $C \subseteq \mathcal{L}$. If $C$ satisfies expansive credibility and closure under double negation, then $C=\{\alpha: \alpha \in A \circledast \alpha\}$.

The next observation illustrates some properties that such a set satisfies whenever $\circledast$ satisfies some of the postulates mentioned in the beginning of Subsection 4.3.

Observation 4.10 Let $A$ be a consistent belief base, $\circledast$ be an operator on $A$ and $C=\{\alpha$ : $\alpha \in A \circledast \alpha\}$.

\begin{tabular}{|c|c|}
\hline If $\circledast$ satisfies & then $C$ satisfies \\
\hline consistency preservation & element consistency \\
\hline strict improvement & single sentence closure \\
\hline disjunctive distribution & disjunctive completeness \\
\hline vacuity & $\begin{array}{c}\text { expansive credibility and credibility } \\
\text { lower bounding }\end{array}$ \\
\hline $\begin{array}{c}\text { consistency preservation, persistence, } \\
\text { relative success and vacuity }\end{array}$ & $\begin{array}{l}\text { satisfies single sentence closure, } \\
\text { disjunctive completeness, revision } \\
\text { credibility, uniform credibility and } \\
\text { credibility of logical equivalents }\end{array}$ \\
\hline $\begin{array}{l}\text { relative success, consistency } \\
\text { preservation, vacuity and uniformity }\end{array}$ & uniform credibility \\
\hline $\begin{array}{c}\text { relative success, consistency } \\
\text { preservation, vacuity and weak } \\
\text { extensionality }\end{array}$ & credibility of logical equivalents \\
\hline $\begin{array}{l}\text { relative success, consistency } \\
\text { preservation, vacuity and uniformity }\end{array}$ & credibility of logical equivalents \\
\hline
\end{tabular}


In the next observation we will see that it is possible to construct an operator $*$ in terms of $\circledast$ and $C=\{\alpha: \alpha \in A \circledast \alpha\}$ and investigate the properties that such an operator satisfies taking into account the properties satisfied by $\circledast$.

Observation 4.11 Let $A$ be a consistent belief base, $\circledast$ be an operator on $A$ and $C=\{\alpha$ : $\alpha \in A \circledast \alpha\}$. Then there exists an operator $*$ on $A$ such that:

(a) If $\circledast$ satisfies relative success, consistency preservation and inclusion, then $*$ is a revision operator and $\circledast$ is the credibility-limited base revision induced by $*$ and $C$.

(b) It holds that:

\begin{tabular}{|c|c|}
\hline If $\circledast$ satisfies & then $*$ satisfies \\
\hline vacuity & vacuity \\
\hline relevance & relevance \\
\hline core-retainment & core-retainment \\
\hline disjunctive elimination & disjunctive elimination \\
\hline $\begin{array}{c}\text { uniformity, relative success, vacuity } \\
\text { consistency preservation }\end{array}$ & uniformity \\
\hline $\begin{array}{c}\text { weak extensionality, relative success, } \\
\text { vacuity, consistency preservation }\end{array}$ & weak extensionality \\
\hline weak relative closure & weak relative closure \\
\hline
\end{tabular}

(c) If $\circledast$ satisfies persistence, relative success and vacuity, then $*$ and $C$ satisfy condition $(\mathbf{C}-*)$.

\section{Axiomatic Characterizations of Different Kinds of Credibility-Limited Base Revision}

In this section we present axiomatic characterizations for several classes of credibility-limited base revision functions. We start by presenting, in Subsection 5.1, a representation theorem for the most general class of credibility-limited base revision operators that we will consider. Afterwards we will consider more specific classes of credibility-limited base revision operators induced by different kinds of revision functions (namely, by partial meet, (smooth) kernel, and basic AGM-generated base revisions) and by different types of sets of credible sentences. In each one of the Subsections $5.2-5.5$ we present a representation theorem for five classes of credibility-limited base revisions all induced by the same kind of revision functions but each one of them with a different type of associated set of credible sentences.

\subsection{Basic Credibility-Limited Base Revision}

In this subsection we present a representation theorem for the most general class of credibility-limited base revision that we will consider. The operators in this class will be designated by basic credibility-limited revisions. 
Definition 5.1 A credibility-limited base revision $\circledast$ on a consistent belief base $A$ induced by a revision operator $*$ and a set $C \subseteq \mathcal{L}$ is a basic credibility-limited revision if and only if $C$ satisfies element consistency.

Theorem 5.2 Let $A$ be a consistent belief base and $\circledast$ be an operator on $A$. Then the following conditions are equivalent:

(a) $\circledast$ satisfies relative success, consistency preservation and inclusion.

(b) $\circledast$ is a basic credibility-limited revision.

In the remainder of this section we will obtain representation theorems for other less general classes of credibility-limited revisions. More precisely, we will consider the credibilitylimited revision operators induced by partial meet base revisions, by (smooth) kernel base revisions and by basic AGM-generated base revisions and, additionally, we will take into account different sets of properties regarding the associated set of credible sentences.

\subsection{Credibility-Limited Partial Meet Base Revisions}

We start this subsection by defining five kinds of operators of credibility-limited base revision induced by a partial meet revision operator $*$ and a set $C$ :

Definition 5.3 A credibility-limited base revision $\circledast$ on a consistent belief base $A$ induced by a partial meet revision $*$ and a set $C \subseteq \mathcal{L}$ is a:

\begin{tabular}{|c|c|}
\hline Designation & $\begin{array}{l}\text { if and only if } C \text { satisfies } \\
\text { element consistency, } \\
\text { expansive credibility and }\end{array}$ \\
\hline $\begin{array}{c}\text { Credibility-limited partial meet revision } \\
(C L P M R)\end{array}$ & uniform credibility \\
\hline $\begin{array}{c}\text { Strictly improving } \\
\text { credibility-limited partial meet revision } \\
(S I-C L P M R)\end{array}$ & $\begin{array}{l}\text { uniform credibility and single } \\
\text { sentence closure }\end{array}$ \\
\hline $\begin{array}{c}\text { Disjunctive distributive } \\
\text { credibility-limited partial meet revision } \\
(D D-C L P M R)\end{array}$ & $\begin{array}{l}\text { uniform credibility and } \\
\text { disjunctive completeness }\end{array}$ \\
\hline $\begin{array}{c}\text { Strictly improving disjunctive } \\
\text { distributive credibility-limited } \\
\text { partial meet revision } \\
(S I+D D-C L P M R)\end{array}$ & $\begin{array}{l}\text { uniform credibility, single } \\
\text { sentence closure and disjunctive } \\
\text { completeness }\end{array}$ \\
\hline $\begin{array}{l}\text { Persistent credibility-limited } \\
\text { partial meet revision } \\
(P-C L P M R)\end{array}$ & condition $(\mathbf{C}-*)$ \\
\hline
\end{tabular}

Throughout this chapter we will sometimes use the acronyms presented in the above definition (and whenever a similar definition is presented) to designate the whole class of the 
corresponding operators (rather than only one of its elements). The designations proposed for each of the classes of revisions proposed in the above definition are connected with the distinguishing postulates of the axiomatic characterizations for these operators that are presented in Theorem 5.4 below.

Theorem 5.4 Let $A$ be a consistent belief base and $\circledast$ an operator on $A$. Then:

\begin{tabular}{|c|c|}
\hline$\circledast$ is $\boldsymbol{a}$ & $\begin{array}{c}\text { if and only if } \circledast \text { satisfies relative success, } \\
\text { consistency preservation, inclusion, vacuity, } \\
\text { uniformity, relevance and }\end{array}$ \\
\hline$C L P M R$ & - \\
\hline$S I-C L P M R$ & strict improvement \\
\hline$D D-C L P M R$ & disjunctive distribution \\
\hline$S I+D D-C L P M R$ & strict improvement and disjunctive distribution \\
\hline$P-C L P M R$ & persistence \\
\hline
\end{tabular}

It is worth noticing that the set $C$ which was used in the proof of the right-to-left part of Theorem 5.4 was the set $C=\{\alpha: \alpha \in A \circledast \alpha\}$. Therefore, it follows immediately from Observations 4.5 and 4.10 that, to the list of properties of $C$ mentioned in each row of the table presented in Definition 5.3, we can add the following ones: credibility lower-bounding, credibility of logical equivalents, negation completeness and closure under double negation. Furthermore, in the case of the last row, besides the properties mentioned above we can also add (to the list of properties of $C$ there presented): single sentence closure, disjunctive completeness, revision credibility and uniform credibility. Therefore, there are several alternative (equivalent) definitions for the classes introduced in Definition 5.3, more precisely several equivalent definitions, but each one with a different set of properties associated to the set $C$. The same is also valid for Definitions 5.6, 5.8 and 5.10.

Next we present examples of credibility-limited revision operators that belong to some of the classes introduced in Definition 5.3 but do not belong to others. These examples allow us to conclude that the classes mentioned in Definition 5.3 are all different from each other.

Example 5.5 Let $A=\{p, q\}$, Cn be purely truth-functional and $*$ be a partial meet revision on $A$ such that $A *(\neg p \vee \neg q)=\{p, \neg p \vee \neg q\}$ and $A *(\neg p \wedge \neg q)=\{\neg p \wedge \neg q\}$. Let $\circledast$ be the operator of credibility-limited base revision induced by $*$ and a set $C$.

(a) Let $C$ be the set defined by the following condition:

$$
\alpha \in C \text { if and only if } \neg \alpha \notin C n(p) \cup C n(q)
$$

Then $C$ satisfies element consistency, expansive credibility, uniform credibility and single sentence closure. ${ }^{17}$ Therefore, by Definition 5.3, $\circledast$ is a CLPMR and a SI$C L P M R$. On the other hand it holds that $\neg p \notin C, \neg q \notin C$ and $\neg p \vee \neg q \in C$ since $p \wedge q \notin C n(p) \cup C n(q)$. Hence $A \circledast \neg p=A \circledast \neg q=A$ but $A \circledast(\neg p \vee \neg q)=A *(\neg p \vee \neg q)=$ $\{p, \neg p \vee \neg q\}$. Thus $\circledast$ does not satisfy disjunctive distribution. Therefore, according to Theorem 5.4, $\circledast$ is not a DD-CLPMR nor a $S I+D D-C L P M R$.

17. See Lemma 1 in the Appendix. 
(b) Let $C$ be the set defined by the following condition:

$$
\alpha \in C \text { if and only if } \neg \alpha \in C n(q) \backslash C n(p)
$$

Then $C$ satisfies element consistency, expansive credibility, uniform credibility and disjunctive completeness. ${ }^{18}$

Therefore, according to Definition 5.3, $\circledast$ is a CLPMR and a DD-CLPMR. On the other hand it holds that $\neg q \in C$ and $\neg p \vee \neg q \notin C$. Thus $A \circledast(\neg p \vee \neg q)=A$ and $A \circledast \neg q=A * \neg q$. Thus $\neg q \in A \circledast \neg q, \vdash \neg q \rightarrow(\neg p \vee \neg q)$ and $\neg p \vee \neg q \notin A \circledast(\neg p \vee \neg q)$, from which it follows that $\circledast$ does not satisfy strict improvement. Therefore, according to Theorem 5.4, $\circledast$ is not a SI-CLPMR nor a SI+DD-CLPMR.

(c) Let $C$ be the set defined by the following condition:

$$
\alpha \in C \text { if and only if } \neg \alpha \notin C n(q)
$$

Then $C$ satisfies element consistency, expansive credibility, uniform credibility, single sentence closure and disjunctive completeness. ${ }^{19}$

Therefore, according to Definition 5.3, $\circledast$ is a SI+DD-CLPMR (and also a SICLPMR, a DD-CLPMR and a CLPMR). On the other hand it holds that $\neg q \notin C$ and $\neg p \vee \neg q \in C$. Thus $A \circledast \neg q=A$ and $A \circledast(\neg p \vee \neg q)=A *(\neg p \vee \neg q)=\{p, \neg p \vee \neg q\}$. Therefore, $A \cap A \circledast \neg q \vdash q$ but $A \cap A \circledast(\neg p \vee \neg q) \forall q$. Thus $\circledast$ does not satisfy persistence. Therefore, according to Theorem 5.4, $\circledast$ is not a P-CLPMR.

\subsection{Credibility-Limited Kernel Base Revisions}

We start this subsection by defining five kinds of operators of credibility-limited base revision induced by a kernel revision $*$ and a set $C$ :

Definition 5.6 A credibility-limited base revision $\circledast$ on a consistent belief base $A$ induced

\begin{tabular}{|c|c|}
\hline Designation & $\begin{array}{l}\text { if and only if } C \text { satisfies } \\
\text { element consistency, } \\
\text { expansive credibility and }\end{array}$ \\
\hline $\begin{array}{c}\text { Credibility-limited kernel revision } \\
(C L K R)\end{array}$ & uniform credibility \\
\hline $\begin{array}{c}\text { Strictly improving } \\
\text { credibility-limited kernel revision } \\
(S I-C L K R)\end{array}$ & $\begin{array}{l}\text { uniform credibility and single } \\
\text { sentence closure }\end{array}$ \\
\hline $\begin{array}{c}\text { Disjunctive distributive } \\
\text { credibility-limited kernel revision } \\
(D D-C L K R)\end{array}$ & $\begin{array}{l}\text { uniform credibility and } \\
\text { disjunctive completeness }\end{array}$ \\
\hline
\end{tabular}
by a kernel revision $*$ and a set $C \subseteq \mathcal{L}$ is a:

Continued on next page

18. See Lemma 2 in the Appendix.

19. See Lemma 3 in the Appendix. 
Continued from previous page

\begin{tabular}{|c|c|}
\hline & if and only if $C$ satisfies: \\
\hline $\begin{array}{c}\text { Strictly improving disjunctive distributive } \\
\text { credibility-limited kernel revision } \\
(S I+D D-C L K R)\end{array}$ & $\begin{array}{c}\text { uniform credibility, single } \\
\text { sentence closure and disjunctive } \\
\text { completeness }\end{array}$ \\
\hline $\begin{array}{c}\text { Persistent credibility-limited } \\
\text { kernel revision } \\
(P-C L K R)\end{array}$ & condition $(\mathbf{C}-*)$ \\
\hline
\end{tabular}

In the following theorem we present axiomatic characterizations for the five kinds of operators of credibility-limited base revision introduced in the above definition.

Theorem 5.7 Let $A$ be a consistent belief base and $\circledast$ an operator on $A$. Then:

\begin{tabular}{|c|c|}
\hline$\circledast$ is $\boldsymbol{a}$ & $\begin{array}{c}\text { if and only if } \circledast \text { satisfies relative success, } \\
\text { consistency preservation, inclusion, vacuity, } \\
\text { uniformity, core-retainment and }\end{array}$ \\
\hline$C L K R$ & - \\
\hline$S I-C L K R$ & strict improvement \\
\hline$D D-C L K R$ & disjunctive distribution \\
\hline$S I+D D-C L K R$ & strict improvement and disjunctive distribution \\
\hline$P-C L K R$ & persistence \\
\hline
\end{tabular}

The difference between the axiomatic characterizations of the classes of credibilitylimited revision induced by partial meet revision operators, presented in the previous subsection, and the ones for the classes of credibility-limited induced by kernel revision operators, presented in Theorem 5.7, is the replacement of relevance by core-retainment, therefore, according to Observation 4.6, every class of credibility-limited revision induced by partial meet revision operators is a subclass of the corresponding class of credibility-limited revision induced by kernel revision operators, i.e., P-CLPMR $\subseteq$ P-CLKR, DD-CLPMR $\subseteq$ $\mathrm{DD}-\mathrm{CLKR}, \mathrm{SI-CLPMR} \subseteq \mathrm{SI}-\mathrm{CLKR}, \mathrm{SI}+\mathrm{DD}-\mathrm{CLPMR} \subseteq \mathrm{SI}+\mathrm{DD}-\mathrm{CLKR}$ and CLPMR $\subseteq$ CLKR.

\subsection{Credibility-Limited Smooth Kernel Base Revisions}

We start this subsection by defining five kinds of operators of credibility-limited base revision induced by a smooth kernel revision operator $*$ and a set $C$ :

Definition 5.8 A credibility-limited base revision $\circledast$ on a consistent belief base $A$ induced by a smooth kernel revision $*$ and a set $C \subseteq \mathcal{L}$ is a: 


\begin{tabular}{|c|c|}
\hline Designation & $\begin{array}{l}\text { if and only if } C \text { satisfies } \\
\text { element consistency, } \\
\text { expansive credibility and }\end{array}$ \\
\hline $\begin{array}{l}\text { Credibility-limited smooth kernel revision } \\
(C L S K R)\end{array}$ & uniform credibility \\
\hline $\begin{array}{c}\text { Strictly improving credibility-limited } \\
\text { smooth kernel revision } \\
(S I-C L S K R)\end{array}$ & $\begin{array}{l}\text { uniform credibility and single } \\
\text { sentence closure }\end{array}$ \\
\hline $\begin{array}{c}\text { Disjunctive distributive credibility-limited } \\
\text { smooth kernel revision } \\
(D D-C L S K R)\end{array}$ & $\begin{array}{l}\text { uniform credibility and } \\
\text { disjunctive completeness }\end{array}$ \\
\hline $\begin{array}{c}\text { Strictly improving disjunctive } \\
\text { distributive credibility-limited } \\
\text { smooth kernel revision } \\
(S I+D D-C L S K R)\end{array}$ & $\begin{array}{c}\text { uniform credibility, single } \\
\text { sentence closure and disjunctive } \\
\text { completeness }\end{array}$ \\
\hline $\begin{array}{c}\text { Persistent credibility-limited smooth kernel } \\
\text { revision } \\
(P-C L S K R)\end{array}$ & condition $(\mathbf{C}-*)$ \\
\hline
\end{tabular}

The following representation theorem axiomatically characterizes five kinds of operators of credibility-limited revisions. All these operators are induced by smooth kernel revisions but each one of them has a different type of associated set of credible sentences.

Theorem 5.9 Let $A$ be a consistent belief base and $\circledast$ an operator on $A$. Then:

\begin{tabular}{|c|c|}
\hline$\circledast$ is $\boldsymbol{a}$ & $\begin{array}{c}\circledast \text { satisfies relative success, consistency } \\
\text { preservation, inclusion, vacuity, uniformity, } \\
\text { core-retainment, weak relative closure and }\end{array}$ \\
\hline$C L S K R$ & - \\
\hline$S I-C L S K R$ & strict improvement \\
\hline$D D-C L S K R$ & disjunctive distribution \\
\hline$S I+D D-C L S K R$ & strict improvement and disjunctive distribution \\
\hline$P-C L S K R$ & persistence \\
\hline
\end{tabular}

The axiomatic characterizations of the classes of credibility-limited revision operators induced by smooth kernel revision operators, presented in Theorem 5.9, can be obtained from the ones presented for credibility-limited revision operators induced by kernel revision operators, presented in Theorem 5.7, by adding weak relative closure. Thus, every class of credibility-limited revision induced by smooth kernel revision operators is a subclass of the corresponding class of credibility-limited revision induced by kernel revision operators. Furthermore, it follows from Theorems 5.4 and 5.9 and Observations 3.5 and 4.6 that every class of credibility-limited revision induced by partial meet revision operators is a subclass of the corresponding class of credibility-limited revision induced by smooth kernel revision operators. That is, it holds that $\mathrm{CLPMR} \subseteq \mathrm{CLSKR} \subseteq \mathrm{CLKR}, \mathrm{SI}-\mathrm{CLPMR} \subseteq \mathrm{SI}-\mathrm{CLSKR} \subseteq$ $\mathrm{SI}-\mathrm{CLKR}, \mathrm{DD}-\mathrm{CLPMR} \subseteq \mathrm{DD}-\mathrm{CLSKR} \subseteq \mathrm{DD}-\mathrm{CLKR}, \mathrm{SI}+\mathrm{DD}-\mathrm{CLPMR} \subseteq \mathrm{SI}+\mathrm{DD}-\mathrm{CLSKR}$ 
$\subseteq \mathrm{SI}+\mathrm{DD}-\mathrm{CLKR}$ and $\mathrm{P}-\mathrm{CLPMR} \subseteq \mathrm{P}-\mathrm{CLSKR} \subseteq \mathrm{P}-\mathrm{CLKR}$. These results will be refined in Observation 6.3 where it is stated that (all of) these set inclusions are in fact strict.

\subsection{Credibility-Limited Basic AGM-Generated Base Revisions}

We start this subsection by defining five kinds of operators of credibility-limited base revision induced by a basic AGM-generated revision operator $*$ and a set $C$ :

Definition 5.10 A credibility-limited base revision $\circledast$ on a consistent belief base $A$ induced by a basic AGM-generated revision * and a set $C \subseteq \mathcal{L}$ is a:

\begin{tabular}{|c|c|}
\hline Designation & $\begin{array}{l}\text { if and only if } C \text { satisfies } \\
\text { element consistency, } \\
\text { expansive credibility and }\end{array}$ \\
\hline $\begin{array}{c}\text { Credibility-limited } \\
\text { basic AGM-generated revision } \\
\text { (CLbAGMR) }\end{array}$ & credibility of logical equivalents \\
\hline $\begin{array}{c}\text { Strictly improving credibility-limited } \\
\text { basic AGM-generated revision } \\
\text { (SI-CLbAGMR) }\end{array}$ & single sentence closure \\
\hline $\begin{array}{c}\text { Disjunctive distributive credibility-limited } \\
\text { basic AGM-generated revision } \\
(D D-C L b A G M R)\end{array}$ & $\begin{array}{l}\text { credibility of logical equivalents } \\
\text { and disjunctive completeness }\end{array}$ \\
\hline $\begin{array}{c}\text { Strictly improving disjunctive } \\
\text { distributive credibility-limited } \\
\text { basic AGM-generated revision } \\
(S I+D D-C L b A G M R)\end{array}$ & $\begin{array}{l}\text { single sentence closure and } \\
\text { disjunctive completeness }\end{array}$ \\
\hline $\begin{array}{c}\text { Persistent credibility-limited } \\
\text { basic AGM-generated revision } \\
(P-C L b A G M R)\end{array}$ & condition $(\mathbf{C}-*)$ \\
\hline
\end{tabular}

In the following representation theorem we axiomatically characterize the five kinds of operators of credibility-limited revisions introduced in the above definition.

Theorem 5.11 Let $A$ be a consistent belief base and $\circledast$ an operator on $A$. Then:

\begin{tabular}{|c|c|}
\hline \multirow{2}{*}{ is $\boldsymbol{a}$} & $\begin{array}{c}\text { if and only if } \circledast \text { satisfies relative success, } \\
\text { consistency preservation, inclusion, vacuity, } \\
\text { weak extensionality, disjunctive elimination } \\
\text { and }\end{array}$ \\
\hline$C L b A G M R$ & - \\
\hline$S I-C L b A G M R$ & strict improvement \\
\hline$D D-C L b A G M R$ & disjunctive distribution \\
\hline$S I+D D-C L b A G M R$ & strict improvement and disjunctive distribution \\
\hline$P-C L b A G M R$ & persistence \\
\hline
\end{tabular}

The axiomatic characterizations of the classes of credibility-limited revision operators induced by AGM-generated base revision operators, presented in Theorem 5.11, can 
be obtained from the ones presented for credibility-limited revision operators induced by partial meet revision operators, presented in Theorem 5.4, by replacing uniformity and relevance by weak extensionality and disjunctive elimination. Thus according to Observations 3.5 and 4.6, every class of credibility-limited revision induced by partial meet revision operators is a subclass of the corresponding class of credibility-limited revision induced by AGM-generated base revision operators. That is, it holds that $\mathrm{CLPMR} \subseteq \mathrm{CLbAGMR}, \mathrm{SI}-\mathrm{CLPMR} \subseteq \mathrm{SI}-\mathrm{CLbAGMR}, \mathrm{DD}-\mathrm{CLPMR} \subseteq \mathrm{DD}-\mathrm{CLb}$ AGMR, $\mathrm{SI}+\mathrm{DD}-\mathrm{CLPMR} \subseteq \mathrm{SI}+\mathrm{DD}-\mathrm{CLbAGMR}$ and $\mathrm{P}-\mathrm{CLPMR} \subseteq \mathrm{P}-\mathrm{CLbAGMR}$. In fact, as it will be stated in Observation 6.5, the inclusions above are (all) strict.

\subsection{Summary of the Results Obtained}

Table 2 summarizes the results obtained in the representation theorems presented in this section. Given a credibility limited revision $\circledast$ the white cells that are on the top of the same column represent the properties that $C$ (the associated set of credible sentences) satisfies. The white cells that are placed on the right of the same row indicates the properties that $\circledast$ satisfies.

\section{Maps Between Credibility-Limited Base Revision Functions}

We start this section by presenting an observation that illustrates the interrelations among classes of credibility-limited base revisions induced by the same type of revision function, but each one of them with a different type of associated set of credible sentences. Through this section we assume that the classes of credibility-limited revision mentioned are formed by operators defined on the same belief base.

Observation 6.1 Let $X$ be any one of the elements of the following set of strings: \{CLPMR, CLSKR, CLKR, CLbAGMR\}. Then:

(a) $P-X \subset S I+D D-X$.

(b) $S I+D D-X \subset D D-X$.

(c) $S I+D D-X \subset S I-X$.

(d) $D D-X \nsubseteq S I-X$ and $S I-X \nsubseteq D D-X$.

(e) $D D-X \subset X$.

(f) $S I-X \subset X$.

In Figure 2 we present a diagram that summarizes the results presented in Observation 6.1 .

In what follows we will study the interrelations among classes of credibility-limited revisions induced by different kinds of revision operators. We start by presenting an example that shows that P-CLSKR $\nsubseteq$ CLPMR and that P-CLKR $\nsubseteq$ CLSKR. $^{20}$

20. Note that having in mind Observation 6.1 it follows from P-CLSKR $\nsubseteq$ CLPMR that CLSKR $\nsubseteq$ CLPMR, SI-CLSKR $\nsubseteq$ SI-CLPMR, DD-CLSKR $\nsubseteq$ DD-CLPMR, SI+DD-CLSKR $\nsubseteq$ SI+DD-CLPMR 


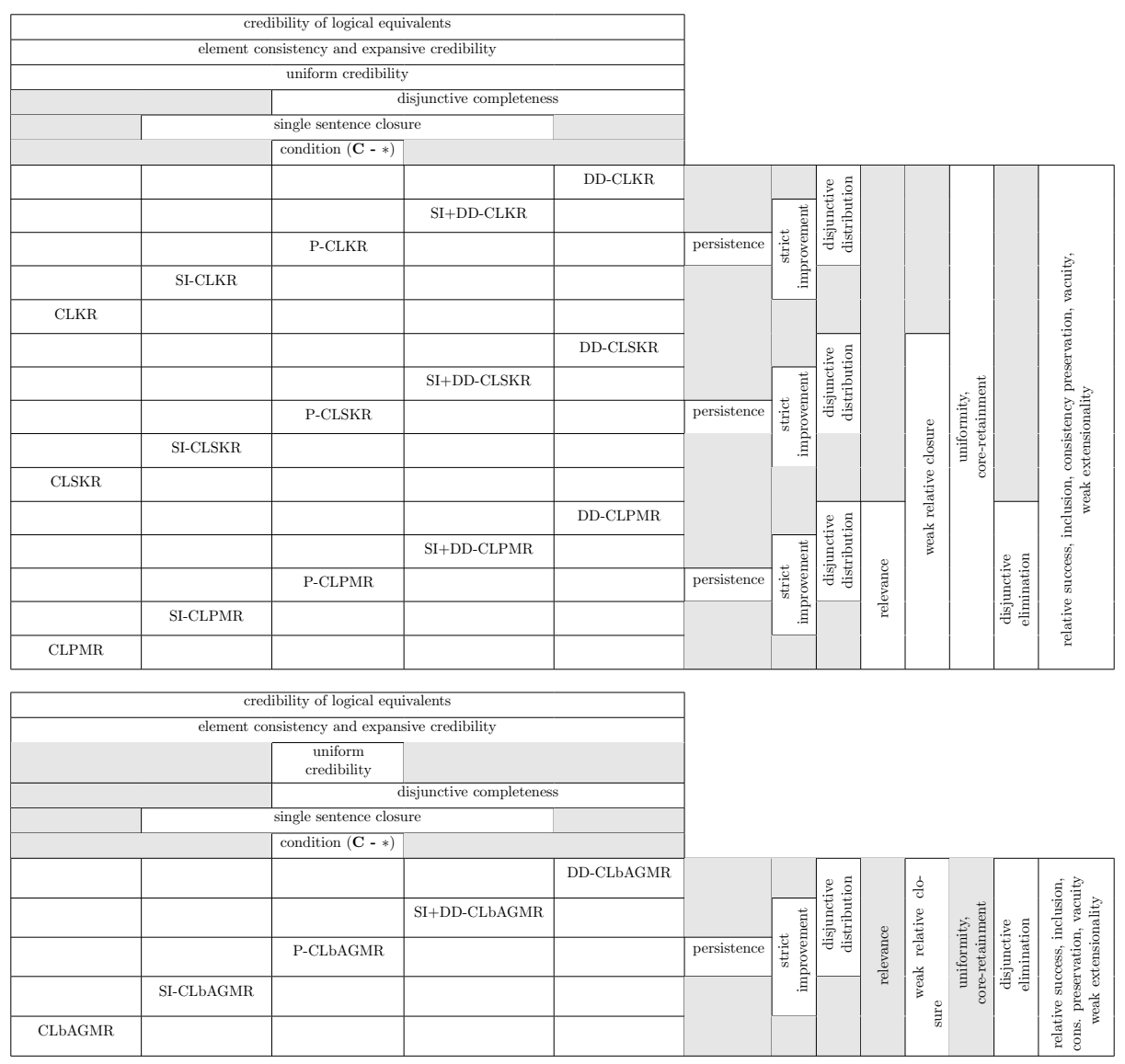

Table 2: Schematic representation of the main postulates satisfied by each one of the twenty classes of credibility limited revisions considered and also of the properties satisfied by the set of credible sentences by which each of those operators is induced. In this table the column headings correspond to properties of $\mathrm{C}$, while the row headings (on the right) correspond to properties of $\circledast$.

Example 6.2 Let $C n$ be purely truth-functional. Let $C=\{\alpha: \neg \alpha \notin C n(\emptyset)\}$. It holds that $C$ satisfies element consistency, expansive credibility and for any revision operator $*$ it holds that $C$ and $*$ satisfy condition $(\mathbf{C}-*)$.

(a) Let $A=\{p, p \vee q, p \leftrightarrow q, r\}$. Hence $A \Perp(p \wedge q)=\{\{p, p \leftrightarrow q\},\{p \vee q, p \leftrightarrow q\}\}$. Let $*$ be the smooth kernel revision based on a smooth incision function $\sigma$ such that: $\sigma(A \Perp(p \wedge q))=\{p, p \leftrightarrow q\}$. Hence $A * \neg(p \wedge q)=(A \backslash \sigma(A \Perp(p \wedge q))) \cup\{\neg(p \wedge q)\}=$ $\{p \vee q, r, \neg(p \wedge q)\}$. Let $\circledast$ be the credibility-limited revision operator induced by $*$ and $C$. Thus $\circledast$ is a P-CLSKR. On the other hand $\neg(p \wedge q) \in C$. Thus $A \circledast \neg(p \wedge q)=$ $A * \neg(p \wedge q)=\{p \vee q, r, \neg(p \wedge q)\}$. Hence $p \in A, p \notin A \circledast \neg(p \wedge q)$, but for any set $A^{\prime}$

and P-CLSKR $\nsubseteq$ P-CLPMR. Analogously, from Observation 6.1, it follows from P-CLKR $\nsubseteq$ CLSKR that CLKR $\nsubseteq$ CLSKR, SI-CLKR $\nsubseteq$ SI-CLSKR, DD-CLKR $\nsubseteq$ DD-CLSKR, SI+DD-CLKR $\nsubseteq$ SI+DDCLSKR and P-CLKR $\nsubseteq$ P-CLSKR. 


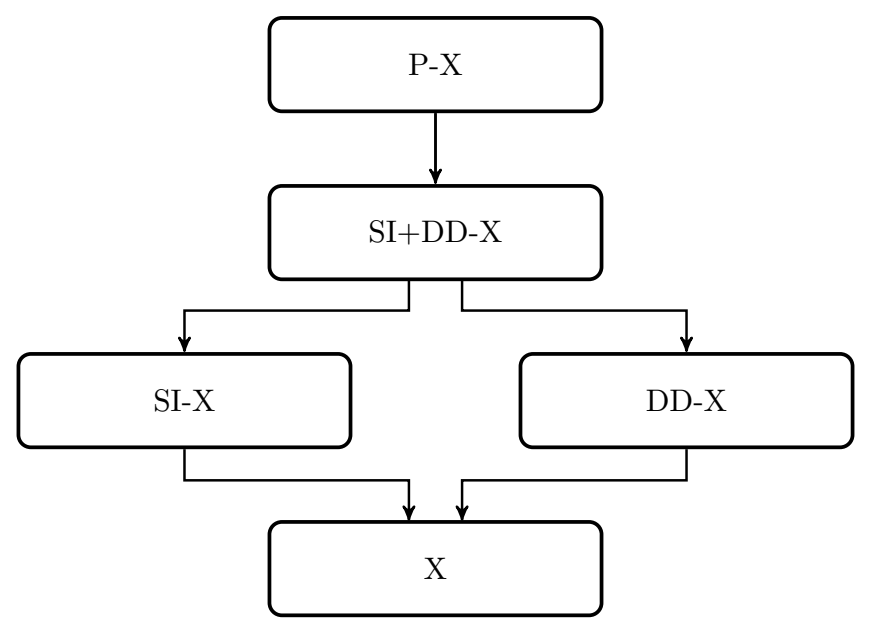

Figure 2: Map among different classes of credibility-limited base revision functions induced by the same kind of revisions. The $X$ must be replaced by one of the following strings CLPMR, CLSKR, CLKR, CLbAGMR. An arrow between two boxes symbolises that the class at the origin of the arrow is a strict subclass of the class at the end of that arrow.

such that $A \circledast \neg(p \wedge q) \subseteq A^{\prime} \subseteq A \cup\{\neg(p \wedge q)\}$ it holds that either $A^{\prime} \vdash \perp$ or $A^{\prime} \cup\{p\} \nvdash \perp$. Hence $\circledast$ does not satisfy relevance. Thus $\circledast$ is not a $C L P M R$.

(b) Let $A=\{p, q, p \vee q, p \rightarrow q\}$. Hence $A \Perp q=\{\{p, p \rightarrow q\},\{p \vee q, p \rightarrow q\},\{q\}\}$. Let $*$ be the kernel revision based on a incision function $\sigma$ such that: $\sigma(A \Perp q)=$ $\{q, p \vee q, p \rightarrow q\}$. Hence $A *(\neg q)=\{p, \neg q\}$. Let $\circledast$ be the credibility-limited revision operator induced by $*$ and $C$. It holds that $\circledast$ is a $P$-CLKR. On the other hand, $\neg q \in C$, thus $A \circledast(\neg q)=A *(\neg q)=\{p, \neg q\}$. Hence $A \cap A \circledast(\neg q)=\{p\}$. Therefore $A \cap C n(A \cap A \circledast(\neg q))=A \cap C n(p)=\{p, p \vee q\} \nsubseteq A \circledast(\neg q)$. Hence $\circledast$ does not satisfy weak relative closure. Hence $\circledast$ is not a $C L S K R$.

The following observation highlights that each one of the classes of CLSKRs that we have considered in Definition 5.8 is subsumed by the corresponding class of CLKRs and, on the other hand, contains the corresponding class of CLPMRs.

\section{Observation 6.3}

(a) $C L P M R \subset C L S K R \subset C L K R$.

(b) $S I-C L P M R \subset S I-C L S K R \subset S I-C L K R$.

(c) $D D-C L P M R \subset D D-C L S K R \subset D D-C L K R$.

(d) $S I+D D-C L P M R \subset S I+D D-C L S K R \subset S I+D D-C L K R$.

(e) $P-C L P M R \subset P-C L S K R \subset P-C L K R$. 
The following example provides a credibility-limited revision that is a P-CLbAGMR but not a CLPMR nor a CLKR. Therefore this example shows that P-CLbAGMR $\nsubseteq$ CLPMR and that P-CLbAGMR $\nsubseteq$ CLKR.

Example 6.4 Let $\mathcal{L}$ be the language that consists only of the two atomic sentences $p$ and $q$ and their truth-functional combinations. Let $C n$ be purely truth-functional. Let $C=\{\alpha$ : $\neg \alpha \notin C n(\emptyset)\}$. It holds that $C$ satisfies element consistency, expansive credibility and for any revision operator $*$ it holds that $C$ and $*$ satisfy condition $(\mathbf{C}-*)$. Let $A=\{p, q\}$. It holds that $C n(A) \perp p=\{C n(p \leftrightarrow q), C n(q)\}$. Let $\star$ be the partial meet revision operator for $C n(A)$ based on a selection function $\gamma$ such that $\gamma(C n(A) \perp p)=\{C n(p \leftrightarrow q)\}$. Hence * is an $A G M$ revision for $C n(A)$ (Alchourrón et al., 1985) and $C n(A) \star(\neg p)=C n(C n(p \leftrightarrow$ $q) \cup\{\neg p\})=C n(\{p \leftrightarrow q, \neg p\})=C n(\neg q \wedge \neg p)$. Let $*$ be an operator defined for all $\alpha$ by $A * \alpha=(C n(A) \star \alpha) \cap(A \cup\{\alpha\})$. Hence * is a basic AGM-generated base revision. Therefore the credibility-limited revision $\circledast$ induced by $*$ and $C$ is a P-CLbAGMR. It holds that $A *(\neg p)=(A \cup\{\neg p\}) \cap C n(\neg q \wedge \neg p)=\{\neg p\}$. From the definition of $C$ it holds that $\neg p \in C$. Thus $A \circledast(\neg p)=A *(\neg p)=\{\neg p\}$. Therefore $q \in A$ and $q \notin A \circledast(\neg p)$, but for any set $A^{\prime} \subseteq A$ it holds that either $A^{\prime} \vdash \neg \neg p$ or $A^{\prime} \cup\{q\} \forall \neg \neg p$. Thus $\circledast$ does not satisfy core-retainment. Hence $\circledast$ is not a CLKR (nor a CLSKR nor a CLPMR).

The following observation highlights that each one of the classes of CLPMRs that we have considered in Subsection 5.2 is strictly contained in the corresponding class of CLbAGMRs.

\section{Observation 6.5}

(a) $C L P M R \subset C L b A G M R$.

(b) $S I-C L P M R \subset S I-C L b A G M R$.

(c) $D D-C L P M R \subset D D-C L b A G M R$.

(d) $S I+D D-C L P M R \subset S I+D D-C L b A G M R$.

(e) $P-C L P M R \subset P-C L b A G M R$.

In the following example we present a credibility-limited base revision that is a P-CLSKR (and consequently a P-CLKR) but not a CLbAGMR.

Example 6.6 Let $C n$ be purely truth-functional. Let $C=\{\alpha: \neg \alpha \notin C n(\emptyset)\}$. It holds that $C$ satisfies element consistency, expansive credibility and for any revision operator $*$ it holds that $C$ and $*$ satisfy condition $(\mathbf{C}-*)$. Let $A=\{p, p \vee q, p \rightarrow q\}$. Hence $A \Perp q=\{\{p, p \rightarrow$ $q\},\{p \vee q, p \rightarrow q\}\}$. Let $*$ be the kernel revision based on a smooth incision function $\sigma$ such that: $\sigma(A \Perp q)=\{p, p \rightarrow q\}$. Hence $A *(\neg q)=(A \backslash \sigma(A \Perp q)) \cup\{\neg q\}=\{p \vee q, \neg q\}$. Let $\circledast$ be the credibility-limited revision operator induced by $*$ and $C$. It holds that $\circledast$ is a P-CLSKR (and a $P$-CLKR). On the other hand, $\neg q \in C$, thus $A \circledast(\neg q)=A *(\neg q)=\{p \vee q, \neg q\}$. Hence $p \in A, p \notin A \circledast(\neg q)$ and $A \circledast(\neg q) \vdash \neg \neg q \vee p$. Therefore, $\circledast$ does not satisfy disjunctive elimination. Thus $\circledast$ is not a $C L b A G M R$. 
The following observation exposes that each one of the classes of CLbAGMRs is not related, in terms of inclusion, neither with the corresponding class of CLSKRs nor with the corresponding class of CLKRs.

\section{Observation 6.7}

(a) CLKR $\nsubseteq C L b A G M R, C L b A G M R \nsubseteq C L K R, C L S K R \nsubseteq C L b A G M R$ and $C L b A G M R \nsubseteq$ CLSKR.

(b) SI-CLKR $\nsubseteq S I-C L b A G M R, S I-C L b A G M R \nsubseteq S I-C L K R, S I-C L S K R \nsubseteq S I-C L b A G M R$ and $S I-C L b A G M R \nsubseteq S I-C L S K R$.

(c) $D D-C L K R \nsubseteq D D-C L b A G M R, D D-C L b A G M R \nsubseteq D D-C L K R, D D-C L S K R \nsubseteq D D-$ $C L b A G M R$ and $D D-C L b A G M R \nsubseteq D D-C L S K R$.

(d) $S I+D D-C L K R \nsubseteq S I+D D-C L b A G M R, S I+D D-C L b A G M R \nsubseteq S I+D D-C L K R, S I+D D-$ $C L S K R \nsubseteq S I+D D-C L b A G M R$ and $S I+D D-C L b A G M R \nsubseteq S I+D D-C L S K R$.

(e) $P-C L K R \nsubseteq P-C L b A G M R, P-C L b A G M R \nsubseteq P-C L K R, P-C L S K R \nsubseteq P-C L b A G M R$ and $P-C L b A G M R \nsubseteq P-C L S K R$.

In Figure 3 we present a diagram that summarizes the results presented in Observations $6.3,6.5$ and 6.7 . The $X$ in this diagram is either a blank space or one of the following strings: SI-, DD-, SI+DD- or P-.

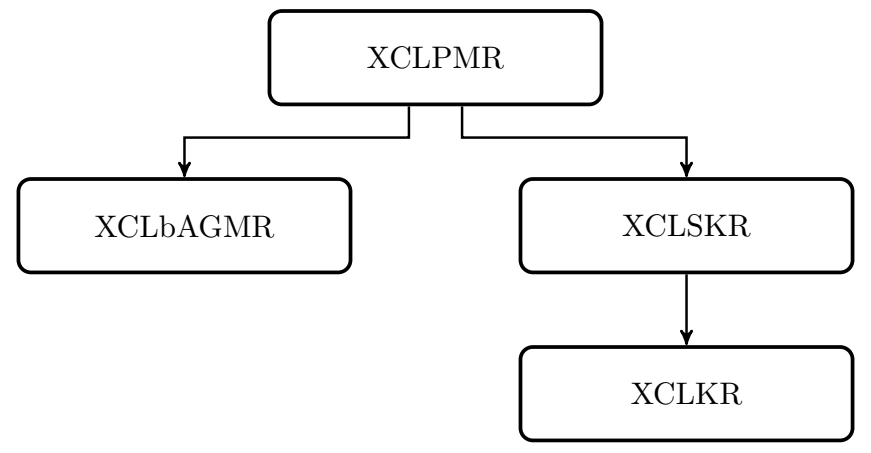

Figure 3: Map among different classes of credibility-limited base revision operators. The $X$ in the diagram must be replaced by a blank space or by an element of the following set of strings: $\{S I-, D D-, S I+D D-, P-\}$.

\section{Conclusion}

Standard revision operators are always successful in the sense that the sentence by which the original belief base is revised is always incorporated in the resulting revised set. However this is not a realistic feature of a belief revision process. When facing new information, an intelligent agent should be able to reject it. 
The basic idea underlying credibility-limited revision is to define a function in two steps. The first step consists of determining if a given belief is credible or not. Then, in a second step the credibility-limited revision operator should:

- leave the set of beliefs unchanged when the belief by which it is revised is considered non-credible;

- behave as a standard revision when revising by a credible belief.

The main contributions of this paper are:

(i) The presentation of several results highlighting some direct relations among the postulates satisfied by a credibility-limited revision function $\circledast$ induced by a (standard) base revision $*$ and a set of credible sentences $C$ and the postulates satisfied by $*$ and the properties of the set $C$.

(ii) The proposal and axiomatic characterization of twenty kinds of credibility-limited base revisions.

(ii) The study of the interrelations among all those classes in terms of (strict) inclusion.

To finish, it is worth mentioning that the representation theorems provided in this paper make it possible to predict the behaviour of any of the proposed functions. We note also that, conversely, by means of the mentioned theorems, for certain sets of properties that are desirable for a credibility-limited revision function, it is possible to identify an explicit construction of a function that will satisfy all those properties.

\section{Acknowledgements}

We wish to thank the Associated Editor who handled this paper and the anonymous referees of the Journal of Artificial Intelligence Research for their very perceptive and pertinent comments on previous versions of the paper that led to a substantial improvement.

This work has been partially supported by Centro de Investigação em Matemática e Aplicações (CIMA) through the grant UIDB/04674/2020 of Fundação para a Ciência e a Tecnologia (FCT), Portugal. EF is partially supported by FCT MCTES IC\&DT Project - AAC 02/SAICT/2017 and NOVA LINCS (UIDB/04516/2020) with the financial support of FCT- Fundação para a Ciência e a Tecnologia, through national funds.

\section{Appendix A. Proofs}

\section{Previous Lemmas}

Lemma 1 Let $A=\{p, q\}, C n$ be purely truth-functional and $C$ be the set defined by the following condition:

$$
\alpha \in C \text { if and only if } \neg \alpha \notin C n(p) \cup C n(q)
$$

Then $C$ satisfies element consistency, expansive credibility, uniform credibility and single sentence closure.

Proof.

Element consistency: Let $\alpha \in C$. Hence $\neg \alpha \notin C n(p) \cup C n(q)$. Thus $\neg \alpha \notin C n(\emptyset)$ (since 
$C n(\emptyset) \subseteq C n(p) \cup C n(q))$. Therefore $\alpha \nvdash \perp$.

Expansive credibility: Assume that $\neg \alpha \notin C$. Hence $\alpha \in C n(p) \cup C n(q)$. Therefore $A \vdash \alpha$.

Uniform credibility: Assume that it holds, for all subsets $A^{\prime} \subseteq A$, that $A^{\prime} \cup\{\alpha\} \vdash \perp$ if and only if $A^{\prime} \cup\{\beta\} \vdash \perp$. Let $\alpha \in C$. Hence $\neg \alpha \notin C n(p) \cup C n(q)$. Thus $\neg \alpha \notin C n(p)$ and $\neg \alpha \notin$ $C n(q)$. Therefore $\{p, \alpha\} \forall \perp$ and $\{q, \alpha\} \forall \perp$. Thus, by hypothesis, $\{p, \beta\} \forall \perp$ and $\{q, \beta\} \forall \perp$. Hence $\neg \beta \notin C n(p)$ and $\neg \beta \notin C n(q)$, from which it follows that $\neg \beta \notin C n(p) \cup C n(q)$. Thus $\beta \in C$. By symmetry of the case it follows that if $\beta \in C$, then $\alpha \in C$. Therefore it holds that $\alpha \in C$ if and only if $\beta \in C$.

Single sentence closure: Let $\alpha \in C$ and $\beta \in C n(\alpha)$. Thus $\neg \alpha \notin C n(p) \cup C n(q)$ and $\{\neg \beta\} \vdash \neg \alpha$. Therefore $\neg \beta \notin C n(p) \cup C n(q)$. Hence $\beta \in C$.

Lemma 2 Let $A=\{p, q\}$, Cn be purely truth-functional and $C$ be the set defined by the following condition:

$$
\alpha \in C \text { if and only if } \neg \alpha \in C n(q) \backslash C n(p)
$$

Then $C$ satisfies element consistency, expansive credibility, uniform credibility and disjunctive completeness.

Proof.

Element consistency: Let $\alpha \in C$. Hence $\neg \alpha \in C n(q) \backslash C n(p)$. It holds that $C n(\emptyset) \subseteq$ $C n(p)$. Thus $\neg \alpha \notin C n(\emptyset)$. Therefore $\alpha \nvdash \perp$.

Expansive credibility: Assume that $\neg \alpha \notin C$. Hence $\neg \neg \alpha \in C n(q) \backslash C n(p)$. Therefore $\alpha \in C n(q)$. Hence $A \vdash \alpha$.

Uniform credibility: Assume that it holds, for all subsets $A^{\prime} \subseteq A$, that $A^{\prime} \cup\{\alpha\} \vdash \perp$ if and only if $A^{\prime} \cup\{\beta\} \vdash \perp$. Let $\alpha \in C$. Hence $\neg \alpha \in C n(q) \backslash C n(p)$. Thus $\neg \alpha \in C n(q)$ and $\neg \alpha \notin C n(p)$. Hence $\{q, \alpha\} \vdash \perp$ and $\{p, \alpha\} \forall \perp$. Thus, by hypothesis, $\{q, \beta\} \vdash \perp$ and $\{p, \beta\} \forall \perp$. Hence $\neg \beta \in C n(q)$ and $\neg \beta \notin C n(p)$, from which it follows that $\beta \in C$. By symmetry of the case it follows that if $\beta \in C$, then $\alpha \in C$. Therefore it holds that $\alpha \in C$ if and only if $\beta \in C$.

Disjunctive completeness: Assume that $\alpha \vee \beta \in C$. Then $\neg(\alpha \vee \beta) \in C n(q) \backslash C n(p)$. Thus $\neg(\alpha \vee \beta) \in C n(q)$ and $\neg(\alpha \vee \beta) \notin C n(p)$. Hence $\neg \alpha \wedge \neg \beta \in C n(q)$ and $\neg \alpha \wedge \neg \beta \notin C n(p)$. Thus $\neg \alpha \in C n(q), \neg \beta \in C n(q)$ and $(\neg \alpha \notin C n(p)$ or $\neg \beta \notin C n(p))$. Therefore $\neg \alpha \in C n(q) \backslash C n(p)$ or $\neg \beta \in C n(q) \backslash C n(p)$. Thus $\alpha \in C$ or $\beta \in C$.

Lemma 3 Let $A=\{p, q\}, C n$ be purely truth-functional and $C$ be the set defined by the following condition:

$$
\alpha \in C \text { if and only if } \neg \alpha \notin C n(q)
$$

Then $C$ satisfies element consistency, expansive credibility, uniform credibility, single sentence closure and disjunctive completeness.

Proof.

Element consistency: Let $\alpha \in C$. Hence $\neg \alpha \notin C n(q)$. Thus $\neg \alpha \notin C n(\emptyset)$. Therefore $\alpha \nvdash \perp$.

Expansive credibility: Assume that $\neg \alpha \notin C$. Hence $\alpha \in C n(q)$. Thus $A \vdash \alpha$. Uniform credibility: Assume that it holds, for all subsets $A^{\prime} \subseteq A$, that $A^{\prime} \cup\{\alpha\} \vdash \perp$ 
if and only if $A^{\prime} \cup\{\beta\} \vdash \perp$. Let $\alpha \in C$. Hence $\neg \alpha \notin C n(q)$, from which it follows that $\{q, \alpha\} \forall \perp$. Thus, by hypothesis, $\{q, \beta\} \not \vdash \perp$. So $\neg \beta \notin C n(q)$. Hence $\beta \in C$. By symmetry of the case it follows that if $\beta \in C$, then $\alpha \in C$. Therefore it holds that $\alpha \in C$ if and only if $\beta \in C$.

Single sentence closure: Let $\alpha \in C$ and $\beta \in C n(\alpha)$. Thus $\neg \alpha \notin C n(q)$ and $\{\neg \beta\} \vdash \neg \alpha$. Therefore $\neg \beta \notin C n(q)$. Hence $\beta \in C$.

Disjunctive completeness: Assume that $\alpha \vee \beta \in C$. Hence $\neg(\alpha \vee \beta) \notin C n(q)$. Thus $\neg \alpha \notin C n(q)$ or $\neg \beta \notin C n(q)$. Thus $\alpha \in C$ or $\beta \in C$.

Lemma 4 Let $A$ be a belief base. Let $*$ be an operator on $A$ such that (for all $\alpha$ ) $A * \alpha=$ $(A \backslash \bigcup(A \Perp \neg \alpha)) \cup\{\alpha\}$, then * satisfies relevance, consistency, success, inclusion, vacuity, weak extensionality, uniformity, core-retainment, disjunctive elimination and weak relative closure.

\section{Proof.}

Let $*$ be an operator on a belief base $A$ defined (for all $\alpha$ ) by $A * \alpha=(A \backslash \bigcup(A \Perp \neg \alpha)) \cup\{\alpha\}$. We will start by showing that $*$ satisfies relevance. Let $\beta \in A$ and $\beta \notin A * \alpha$. Thus $\beta \in \bigcup(A \Perp \neg \alpha)$. Hence there exists $Y \in A \Perp \neg \alpha$ such that $\beta \in Y$. Let $X=Y \backslash\{\beta\} \cup(A \backslash \bigcup(A \Perp \neg \alpha)) \cup\{\alpha\}$. Hence $X \subseteq A \cup\{\alpha\}, A * \alpha=(A \backslash \bigcup(A \Perp \neg \alpha)) \cup\{\alpha\} \subseteq X$ and $X \cup\{\beta\} \vdash \perp$ (since $X \cup\{\beta\} \vdash \neg \alpha$ and $\alpha \in X$ ). It remains to prove that $X \forall \perp$. Assume by reductio ad absurdum that $X \vdash \perp$. Hence, by deduction it follows that $X^{\prime}=Y \backslash\{\beta\} \cup(A \backslash \bigcup(A \Perp \neg \alpha)) \vdash \neg \alpha$. It follows by compactness that there exists a finite subset $H=\left\{\gamma_{1}, \ldots, \gamma_{n}\right\}$ of $X^{\prime}$ such that $H \vdash \neg \alpha$. Where $\gamma_{1}, \ldots, \gamma_{k} \in Y \backslash\{\beta\}$ and $\gamma_{k+1}, \ldots, \gamma_{n} \in A \backslash \bigcup(A \Perp \neg \alpha)$, for some $1 \leq k<n$. Hence $\left\{\gamma_{1}, \ldots, \gamma_{k}\right\} \cup\left\{\gamma_{k+1}, \ldots, \gamma_{n}\right\} \vdash \neg \alpha$ but $\left\{\gamma_{1}, \ldots, \gamma_{k}\right\} \forall \neg \alpha$. Thus there is some inclusion-minimal subset $W$ of $H$ such that $W \vdash \neg \alpha$ but no proper set of $W$ implies $\neg \alpha$. Hence $W \in A \Perp \neg \alpha$. On the other hand, since $\left\{\gamma_{1}, \ldots, \gamma_{k}\right\} \forall \neg \alpha, W$ contains at least one of the $\gamma_{i} \in\left\{\gamma_{k+1}, \ldots, \gamma_{n}\right\}$. Contradiction since $\left\{\gamma_{k+1}, \ldots, \gamma_{n}\right\} \subseteq A \backslash \bigcup(A \Perp \neg \alpha)$. Hence $*$ satisfies relevance.

On the other hand, by definition of kernel revision, $*$ is a kernel revision. Hence, by Observations 2.11, * satisfies consistency, success, inclusion, uniformity and core-retainment. On the other hand, by Observation 3.5, * satisfies vacuity, weak extensionality, disjunctive elimination and weak relative closure.

\section{Proofs}

\section{Proof of Theorem 3.2.}

(Construction-to-postulates)

Let $*$ be an operator of smooth kernel revision on $A$. It follows from Observation 2.11 that * satisfies consistency, success, inclusion, uniformity and core-retainment. It remains to prove that $*$ satisfies weak relative closure. Since $*$ is a kernel revision operator it follows that $*$ is based on a incision function $\sigma$ such that for all sentences $\alpha$ :

$$
A * \alpha=(A \backslash \sigma(A \Perp \neg \alpha)) \cup\{\alpha\}
$$


On the other hand, $\sigma$ is smooth. Hence it holds for all subsets $A^{\prime}$ of $A$ that if $A^{\prime} \vdash \beta$ and $\beta \in \sigma(A \Perp \alpha)$ then $A^{\prime} \cap \sigma(A \Perp \alpha) \neq \emptyset$.

Assume that $\beta \notin A * \alpha$. By $*$ success it follows that $\beta \neq \alpha$. We intend to prove that $\beta \notin A \cap C n(A \cap A * \alpha)$. It follows trivially if $\beta \notin A$. Consider now that $\beta \in A$. Hence, by definition of $*, \beta \in \sigma(A \Perp \neg \alpha)$. Assume by reductio ad absurdum that $\beta \in A \cap C n(A \cap A * \alpha)$. Then $((A \backslash \sigma(A \Perp \neg \alpha)) \cup\{\alpha\}) \cap A \vdash \beta$. Thus $((A \backslash \sigma(A \Perp \neg \alpha)) \cup(A \cap\{\alpha\})) \vdash \beta$. We will consider two cases:

Case 1) $\alpha \notin A$. Hence $A \backslash \sigma(A \Perp \neg \alpha) \vdash \beta$. Let $X=A \backslash \sigma(A \Perp \neg \alpha)$. Hence $X \subseteq A, X \vdash \beta$, $\beta \in \sigma(A \Perp \neg \alpha)$ and $X \cap \sigma(A \Perp \neg \alpha)=\emptyset$. Which contradicts the fact that $\sigma$ is smooth.

Case 2) $\alpha \in A$. Hence, by deduction, $A \backslash \sigma(A \Perp \neg \alpha) \vdash \alpha \rightarrow \beta$. Suppose that $\alpha \in \sigma(A \Perp \neg \alpha)$. Hence there exists $Y \subseteq A \Perp \neg \alpha$ such that $\alpha \in Y$. Let $Y^{\prime}=Y \backslash\{\alpha\}$. Thus $Y^{\prime} \forall \neg \alpha$ but $Y^{\prime} \cup\{\alpha\} \vdash \neg \alpha$. Hence by deduction, $Y^{\prime} \vdash \alpha \rightarrow \neg \alpha$. Contradiction, since $\vdash \neg \alpha \leftrightarrow(\alpha \rightarrow \neg \alpha)$. Thus $\alpha \notin \sigma(A \Perp \neg \alpha)$, from which it follows that $\alpha \in A \backslash \sigma(A \Perp \neg \alpha)$ and consequently that $A \backslash \sigma(A \Perp \neg \alpha) \vdash \beta$. The rest of the proof for this case follows as in the previous one.

(Postulates-to-construction)

Let $*$ be an operator that satisfies all the postulates listed in the observation. Let $\sigma(A \Perp \neg \alpha)=A \backslash(A \cap(A * \alpha))$. This is the same construction that is used in the proof of Observation 2.11 that was presented by Wassermann (2000, Proof of Theorem 5.2.14). Hence $\sigma$ is an incision function for $A$ and $A * \alpha=(A \backslash \sigma(A \Perp \neg \alpha)) \cup\{\alpha\}$. It remains to show that $\sigma$ is smooth. Let $A^{\prime}$ be a subset of $A$ such that $A^{\prime} \vdash \beta$ and $\beta \in \sigma(A \Perp \neg \alpha)$. We intend to prove that $A^{\prime} \cap \sigma(A \Perp \neg \alpha) \neq \emptyset$.

Assume by reductio ad absurdum that $A \cap A * \alpha \vdash \beta$. From $\beta \in \sigma(A \Perp \neg \alpha)$ it follows that $\beta \in A$. Thus by $*$ relative closure it follows that $\beta \in A * \alpha$. Contradiction, since $\beta \in \sigma(A \Perp \neg \alpha)=A \backslash(A \cap(A * \alpha))$. Therefore $A \cap A * \alpha \forall \beta$. From $A^{\prime} \vdash \beta$ it follows that $A^{\prime} \nsubseteq A \cap A * \alpha$. Hence there is some sentence $\delta \in A^{\prime} \backslash(A \cap A * \alpha)$. Thus $\delta \in \sigma(A \Perp \neg \alpha)$. Therefore $\delta \in A^{\prime} \cap \sigma(A \Perp \neg \alpha)$, from which it follows that $A^{\prime} \cap \sigma(A \Perp \neg \alpha) \neq \emptyset$.

\section{Proof of Theorem 3.4.}

(Construction-to-postulates)

Let $A * \alpha=(C n(A) \star \alpha) \cap(A \cup\{\alpha\})$ and $\star$ be a basic AGM revision for $C n(A)$. Hence $\star$ satisfies success, inclusion, vacuity, consistency, extensionality and closure. We will now prove that $*$ satisfies consistency, success, inclusion, vacuity, weak extensionality and disjunctive elimination.

Success: Follows trivially by $*$ definition and $\star$ success.

Inclusion: Follows trivially by $*$ definition.

Vacuity: Let $A \nvdash \neg \alpha$ and $\beta \in A \cup\{\alpha\}$. If $\beta=\alpha$, then $\beta \in A * \alpha$ by definition of $*$ and $\star$ success. Assume now that $\beta \neq \alpha$. Then $\beta \in A$. Hence, by $\star$ vacuity $\operatorname{Cn}(\operatorname{Cn}(A) \cup$ $\{\alpha\}) \subseteq C n(A) \star \alpha$. On the other hand, ${ }^{21} C n(C n(A) \cup\{\alpha\})=C n(A \cup\{\alpha\})$ therefore $A \cup\{\alpha\}=C n(A \cup\{\alpha\}) \cap(A \cup\{\alpha\}) \subseteq(C n(A) \star \alpha) \cap(A \cup\{\alpha\})=A * \alpha$.

Weak extensionality: Let $\vdash \alpha \leftrightarrow \beta$. Then $A \cap A * \alpha=A \cap((C n(A) \star \alpha) \cap(A \cup\{\alpha\}))=$ $A \cap(C n(A) \star \alpha)$. Thus, by $\star$ extensionality, $A \cap A * \alpha=A \cap(C n(A) \star \beta)$. Hence, by definition of $*$, it follows that $A \cap A * \alpha=A \cap A * \beta$.

21. $C n(A \cup B)=C n(A \cup C n(B))$ (Hansson, 1999b). 
Disjunctive elimination: Let $\beta \in A$ and $\beta \notin A * \alpha$. Then, by definition of $*, \beta \notin$ $(C n(A) \star \alpha) \cap(A \cup\{\alpha\})$. Hence $\beta \notin C n(A) \star \alpha$. On the other hand, by $\star$ success, $\alpha \in C n(A) \star \alpha$. Thus, by $\star$ closure, $C n(A) \star \alpha \forall \neg \alpha \vee \beta$. Therefore, by definition of $*$ it follows that $A * \alpha \forall \neg \alpha \vee \beta$.

Consistency: Let $\alpha \nvdash \perp$. By $\star$ consistency it follows that $C n(A) \star \alpha \nvdash \perp$. Hence $A * \alpha \nvdash \perp$. (Postulates-to-construction)

Let $*$ be an operator on $A$ that satisfies consistency, success, inclusion, vacuity, weak extensionality and disjunctive elimination. Let $\star$ be an operator on $C n(A)$ defined, for all $\alpha \in \mathcal{L}$, as follows:

$$
C n(A) \star \alpha=C n(A * \alpha)
$$

We must prove that:

a) * satisfies success, consistency, extensionality, inclusion, vacuity and closure;

b) $A * \alpha=(C n(A) \star \alpha) \cap(A \cup\{\alpha\})$.

Proof of a) Closure follows trivially from $\star$ definition. Success, consistency and inclusion follow trivially from $\star$ definition and $*$ success, consistency and inclusion respectively.

Vacuity: Let $\neg \alpha \notin C n(A)$. By * vacuity it follows that $A \cup\{\alpha\} \subseteq A * \alpha$. Thus $C n(C n(A) \cup\{\alpha\})=C n(A \cup\{\alpha\}) \subseteq C n(A * \alpha)=C n(A) \star \alpha$.

Extensionality: Let $\vdash \alpha \leftrightarrow \beta$. It follows, by $*$ weak extensionality, that $A \cap A * \alpha=A \cap A * \beta$. We will prove by double inclusion that $C n(A) \star \alpha=C n(A) \star \beta$.

We will start by proving that $A * \alpha=(A \cap A * \alpha) \cup\{\alpha\}$.

$(A \cap A * \alpha) \cup\{\alpha\}=(A \cup\{\alpha\}) \cap(A * \alpha \cup\{\alpha\})=A * \alpha$ (the last equality follows from * success and inclusion).

Let $\delta \in C n(A) \star \alpha$. Then $A * \alpha \vdash \delta$. Thus $(A \cap A * \alpha) \cup\{\alpha\} \vdash \delta$. Therefore, by deduction $(A \cap A * \alpha) \vdash \alpha \rightarrow \delta$. It follows, from $\vdash \alpha \leftrightarrow \beta$ and $*$ weak extensionality, that $(A \cap A * \beta) \vdash \beta \rightarrow \delta$. Hence $(A \cap A * \beta) \cup\{\beta\} \vdash \delta$. Therefore $A * \beta \vdash \delta$. Hence $\delta \in C n(A) \star \beta$. Thus $C n(A) \star \alpha \subseteq C n(A) \star \beta$. By symmetry of the case it holds that $C n(A) \star \beta \subseteq C n(A) \star \alpha$. Therefore $C n(A) \star \alpha=C n(A) \star \beta$.

Proof of b) That $A * \alpha \subseteq(C n(A) \star \alpha) \cap(A \cup\{\alpha\})$, follows from $\star$ definition and * inclusion. Let $\delta \in(C n(A) \star \alpha) \cap(A \cup\{\alpha\})$. If $\delta=\alpha$, then it follows from * success that $\delta \in A * \alpha$. Assume now that $\delta \neq \alpha$. Hence $\delta \in A$ and $A * \alpha \vdash \delta$. From the latter it follows that $A * \alpha \vdash \neg \alpha \vee \delta$. Hence, by disjunctive elimination, it follows that $\delta \in A * \alpha$. Thus $(C n(A) \star \alpha) \cap(A \cup\{\alpha\}) \subseteq A * \alpha$. Therefore $(C n(A) \star \alpha) \cap(A \cup\{\alpha\})=A * \alpha$.

\section{Proof of Observation 3.5.}

(a) Trivial

(b) Let $\beta \in A \cap C n(A \circledast \alpha \cap A)$ and assume by reductio ad absurdum that $\beta \notin A \circledast \alpha$. Then, by disjunctive elimination, $A \circledast \alpha \forall \neg \alpha \vee \beta$. On the other hand, from $\beta \in C n(A \circledast \alpha \cap A)$ it follows by monotony of $C n$ that $A \circledast \alpha \vdash \beta$ then $A \circledast \alpha \vdash \neg \alpha \vee \beta$. Contradiction. Therefore, $\beta \in A \circledast \alpha$. Hence $A \cap C n(A \circledast \alpha \cap A) \subseteq A \circledast \alpha$.

(c) Let $A$ be a belief base and $\circledast$ be an operator on $A$ that satisfies relative success and relevance. Let $\beta \in A$ and $\beta \notin A \circledast \alpha$. It follows by relative success that $\alpha \in A \circledast \alpha$. On the other hand, by relevance, there exists some $A^{\prime}$ such that $A \circledast \alpha \subseteq A^{\prime} \subseteq$ 
$A \cup\{\alpha\}, A^{\prime} \forall \perp$ but $A^{\prime} \cup\{\beta\} \vdash \perp$. Since $\alpha \in A \circledast \alpha$ it holds that $\alpha \in A^{\prime}$. Let $B=A^{\prime} \backslash\{\alpha\}$. Hence $B \subseteq A$. On the other hand, since $A^{\prime} \forall \perp$ it follows that $B \cup\{\alpha\} \nvdash \perp$. Thus $B \forall \neg \alpha$. From $A^{\prime} \cup\{\beta\} \vdash \perp$ it follows that $(B \cup\{\alpha\}) \cup\{\beta\} \vdash \perp$. Hence, by deduction $B \cup\{\beta\} \vdash \neg \alpha$.

To prove that $\circledast$ satisfies disjunctive elimination let $\beta \in A$ and $\beta \notin A \circledast \alpha$. By success it follows that $\alpha \in A \circledast \alpha$. Assume by reductio ad absurdum that $A \circledast \alpha \vdash \neg \alpha \vee \beta$. Thus $A \circledast \alpha \vdash \beta$. On the other hand, by relevance, there is some set $A^{\prime}$ such that $A \circledast \alpha \subseteq A^{\prime} \subseteq A \cup\{\alpha\}$ and $A^{\prime} \not \forall \perp$ but $A^{\prime} \cup\{\beta\} \vdash \perp$. Contradiction, since $A^{\prime} \vdash \beta$. Therefore, $A \circledast \alpha \forall \neg \alpha \vee \beta$.

(d) Let $A \nvdash \neg \alpha$. Assume by reductio ad absurdum that $A \cup\{\alpha\} \nsubseteq A \circledast \alpha$. By success it follows that $\alpha \in A \circledast \alpha$. Hence, there exists some $\beta$ such that $\beta \in A$ and $\beta \notin A \circledast \alpha$. Thus, by core-retainment, there exists $A^{\prime} \subseteq A$ such that $A^{\prime} \forall \neg \alpha$ and $A^{\prime} \cup\{\beta\} \vdash \neg \alpha$. Contradiction, since $A^{\prime} \cup\{\beta\} \subseteq A$. Therefore, $A \cup\{\alpha\} \subseteq A \circledast \alpha$.

\section{Proof of Observation 4.3.}

Let $A$ be a belief base and $\circledast$ be a credibility-limited base revision induced by a revision operator $*$ on $A$ and a set $C \subseteq \mathcal{L}$ that satisfies closure under double negation.

Assume first that $C$ satisfies strong expansive credibility. We will show that $C$ satisfies expansive credibility and that $C$ and $*$ are related through condition $(\mathbf{C}-*)$.

Expansive credibility: Let $\neg \alpha \notin C$. By strong expansive credibility it holds that $A \cap A \circledast \beta \vdash \alpha$. Thus $A \vdash \alpha$.

Condition ( $\mathbf{C}-*$ ): Let $\alpha \notin C$ and $\beta \in C$. Then $A \circledast \beta=A * \beta$. On the other hand, by strong expansive credibility, it holds that $A \cap A \circledast \beta \vdash \neg \alpha$. Thus $A \cap A * \beta \vdash \neg \alpha$.

Assume now that $C$ satisfies expansive credibility and that $C$ and $*$ are related through condition $(\mathbf{C}-*)$. Let $\alpha \notin C$. We will consider two cases:

Case 1) $\beta \in C$. Hence, by condition $(\mathbf{C}-*), A \cap A * \beta \vdash \neg \alpha$. Thus $A \cap A \circledast \beta \vdash \neg \alpha$.

Case 2) $\beta \notin C$. It holds that $A \circledast \beta \cap A=A$. On the other hand, from $\alpha \notin C$, it follows by closure under double negation that $\neg \neg \alpha \notin C$. Thus, by expansive credibility, it follows that $A \vdash \neg \alpha$. Therefore $A \cap A \circledast \beta \vdash \neg \alpha$.

\section{Proof of Observation 4.4.}

Assume first that it holds that $C n(\emptyset) \subseteq C$. Hence $(\neg \alpha \vee \alpha) \in C$. Therefore, by disjunctive completeness it follows that $\neg \alpha \in C$ or $\alpha \in C$.

Assume now that $C$ satisfies negation completeness. Let $\alpha \in \mathcal{L}$ be such that $\vdash \alpha$. Hence $\neg \alpha \vdash \perp$. Therefore, by element consistency, $\neg \alpha \notin C$, from which it follows by negation completeness that $\alpha \in C$.

\section{Proof of Observation 4.5.}

(a) Let $\vdash \alpha \leftrightarrow \beta$. If $\alpha \in C$, then by single sentence closure $\beta \in C$. By symmetry of the case it follows that is $\beta \in C$, then $\alpha \in C$. Thus $\alpha \in C$ if and only if $\beta \in C$.

(b) Let $\vdash \alpha \leftrightarrow \beta$. It follows trivially by uniform credibility, that $\alpha \in C$ if and only if $\beta \in C$. 
(c) Let $A \nvdash \perp$ and $\neg \alpha \notin C$. Then by expansive credibility it follows that $A \vdash \alpha$. Therefore by credibility lower bounding $\alpha \in C$.

(d) Let $\alpha \in C n(\emptyset)$. Then $\neg \alpha \vdash \perp$. From which it follows by element consistency that $\neg \alpha \notin C$. Therefore, by negation completeness, $\alpha \in C$.

(e) Trivial.

\section{Proof of Observation 4.6.}

Let $A$ be a belief base and $\circledast$ be an operator on $A$ that satisfies relevance and relative success.

To prove that $\circledast$ satisfies disjunctive elimination, let $\beta \in A$ and $\beta \notin A \circledast \alpha$. It follows by relative success that $\alpha \in A \circledast \alpha$. Assume by reductio ad absurdum that $A \circledast \alpha \vdash \neg \alpha \vee \beta$. Hence $A \circledast \alpha \vdash \beta$. On the other hand, by relevance, there exists some $A^{\prime}$ such that $A \circledast \alpha \subseteq A^{\prime} \subseteq A \cup\{\alpha\}, A^{\prime} \nvdash \perp$ but $A^{\prime} \cup\{\beta\} \vdash \perp$. Contradiction, since every set that contains $A \circledast \alpha$ implies $\beta$.

To prove that $\circledast$ satisfies core-retainment, let $\beta \in A$ and $\beta \notin A \circledast \alpha$. It follows by relative success that $\alpha \in A \circledast \alpha$. On the other hand, by relevance, there exists some $A^{\prime}$ such that $A \circledast \alpha \subseteq A^{\prime} \subseteq A \cup\{\alpha\}, A^{\prime} \nvdash \perp$ but $A^{\prime} \cup\{\beta\} \vdash \perp$. Since $\alpha \in A \circledast \alpha$ it holds that $\alpha \in A^{\prime}$. Let $B=A^{\prime} \backslash\{\alpha\}$. Hence $B \subseteq A$. On the other hand, since $A^{\prime} \nvdash \perp$ it follows that $B \cup\{\alpha\} \forall \perp$. Thus $B \forall \neg \alpha$. From $A^{\prime} \cup\{\beta\} \vdash \perp$ it follows that $(B \cup\{\alpha\}) \cup\{\beta\} \vdash \perp$. Hence, by deduction $B \cup\{\beta\} \vdash \neg \alpha$.

\section{Proof of Observation 4.7.}

Assume that $A \not \forall \perp$.

Disjunctive distribution Let $\alpha \notin A \circledast \alpha$ and $\beta \notin A \circledast \beta$. By relative success it follows that $A \circledast \alpha=A$ and $A \circledast \beta=A$. If $A \nvdash \neg \alpha$, then by vacuity $A \cup\{\alpha\} \subseteq A \circledast \alpha$, from which it follows that $\alpha \in A \circledast \alpha$. Contradiction. Hence $A \vdash \neg \alpha$. By symmetry of the case it follows that $A \vdash \neg \beta$. Thus $A \cap A \circledast \alpha \vdash \neg \alpha$ and $A \cap A \circledast \beta \vdash \neg \beta$. Hence, by persistence, $A \cap A \circledast(\alpha \vee \beta) \vdash \neg \alpha$ and $A \cap A \circledast(\alpha \vee \beta) \vdash \neg \beta$. Therefore $A \cap A \circledast(\alpha \vee \beta) \vdash \neg \alpha \wedge \neg \beta$. Thus $A \cap A \circledast(\alpha \vee \beta) \vdash \neg(\alpha \vee \beta)$. Hence by monotony $A \circledast(\alpha \vee \beta) \vdash \neg(\alpha \vee \beta)$. It follows, by consistency preservation, that $\alpha \vee \beta \notin A \circledast(\alpha \vee \beta)$.

Strict improvement Let $\alpha \in A \circledast \alpha$ and $\vdash \alpha \rightarrow \beta$. By relative success it follows that $\beta \in A \circledast \beta$ or $A \circledast \beta=A$. In the first case we are done. Consider now that $A \circledast \beta=A$. Assume by reductio ad absurdum that $A \vdash \neg \beta$. Hence $A \cap A \circledast \beta \vdash \neg \beta$. Thus, by persistence, $A \cap A \circledast \alpha \vdash \neg \beta$. Hence $A \circledast \alpha \vdash \neg \beta$. On the other hand, from $\alpha \in A \circledast \alpha$ and $\vdash \alpha \rightarrow \beta$, it follows that $A \circledast \alpha \vdash \beta$. Therefore, $A \circledast \alpha \vdash \perp$, which contradicts consistency preservation. Hence $A \nvdash \neg \beta$. Vacuity holds, therefore $\beta \in A \circledast \beta$.

Regularity Let $A \circledast \alpha \vdash \beta$. By relative success it follows that $\beta \in A \circledast \beta$ or $A \circledast \beta=A$. In the first case we are done. Consider now that $A \circledast \beta=A$. Assume by reductio ad absurdum that $A \vdash \neg \beta$. Hence $A \cap A \circledast \beta \vdash \neg \beta$. Thus, by persistence, $A \cap A \circledast \alpha \vdash \neg \beta$. Hence $A \circledast \alpha \vdash \neg \beta$. This contradicts consistency preservation. Hence $A \nvdash \neg \beta$. Vacuity holds, therefore $\beta \in A \circledast \beta$.

Proof of Observation 4.8.

Let $A$ be a belief base, $C \subseteq \mathcal{L}$, and $\circledast$ be a credibility-limited base revision induced by a 
revision operator $*$ and $C$.

Thus,

$$
A \circledast \alpha= \begin{cases}A * \alpha & \text { if } \alpha \in C \\ A & \text { otherwise }\end{cases}
$$

where, by Definition 2.3, * is an operator on $A$ that satisfies success, consistency and inclusion.

(a) That $\circledast$ satisfies inclusion and relative success follows trivially by its definition and $*$ inclusion and $*$ success, respectively.

Let $*$ be an operator on $A$ that satisfies relevance. Let $\beta \in A$ and $\beta \notin A \circledast \alpha$. Hence $A \circledast \alpha=A * \alpha$, and it follows trivially from $*$ relevance that $\circledast$ satisfies relevance.

Let $*$ be an operator on $A$ that satisfies core-retainment. Let $\beta \in A$ and $\beta \notin A \circledast \alpha$. Hence $A \circledast \alpha=A * \alpha$, and it follows trivially from $*$ core-retainment that $\circledast$ satisfies core-retainment.

Let $*$ be an operator on $A$ that satisfies disjunctive elimination. Let $\beta \in A$ and $\beta \notin A \circledast \alpha$. Hence $A \circledast \alpha=A * \alpha$, and it follows trivially from $*$ disjunctive elimination that $\circledast$ satisfies disjunctive elimination.

Let $*$ be an operator on $A$ that satisfies weak relative closure. If $\alpha \in C$, then $A \circledast \alpha=$ $A * \alpha$. Thus, by $*$ weak relative closure it follows that $A \cap C n(A \cap A \circledast \alpha) \subseteq A \circledast \alpha$. If $\alpha \notin C$, then $A \circledast \alpha=A$. Thus $A \cap C n(A \cap A \circledast \alpha)=A=A \circledast \alpha$.

(b) Let $C$ be a set that satisfies element consistency and $\alpha \in \mathcal{L}$. We will show that $\circledast$ satisfies consistency preservation. Let $A \not \perp$. It follows trivially if $\alpha \notin C$. Assume now that $\alpha \in C$. Then $A \circledast \alpha=A * \alpha$. On the other hand, by element consistency, $\alpha \nvdash \perp$. Hence, by $*$ consistency $A \circledast \alpha \forall \perp$.

(c) Let $C$ be a set that satisfies uniform credibility and * a revision operator that satisfies uniformity. Let it be the case that for all subsets $A^{\prime} \subseteq A, A^{\prime} \cup\{\alpha\} \vdash \perp$ if and only if $A^{\prime} \cup\{\beta\} \vdash \perp$. By uniform credibility $\alpha \in C$ if and only if $\beta \in C$. If $\alpha \in C$, then $\beta \in C$. The rest follows from $*$ uniformity.

If $\alpha \notin C$, then $\beta \notin C$. Thus $A \cap A \circledast \alpha=A=A \cap A \circledast \beta$.

(d) We start by noticing that, according to Observation 4.5, if $C$ satisfies single sentence closure, then it also satisfies credibility of logical equivalents. Let $C$ be a set that satisfies credibility of logical equivalents and $*$ a revision operator that satisfies weak extensionality. We intend to prove that $\circledast$ satisfies weak extensionality. Let $\vdash \alpha \leftrightarrow \beta$. By credibility of logical equivalents $\alpha \in C$ if and only if $\beta \in C$. If $\alpha \in C$, then $\beta \in C$. The rest follows from $*$ weak extensionality. If $\alpha \notin C$, then $\beta \notin C$, from which it follows that $A \circledast \alpha=A \circledast \beta=A$. Thus $A \cap A \circledast \alpha=A \cap A \circledast \beta$. 
(e) We start by noticing that, according to Observation 4.5, if $C$ satisfies either credibility of logical equivalents or uniform credibility or single sentence closure, then it also satisfies closure under double negation. Let $C$ be a set that satisfies expansive credibility and closure under double negation and $*$ a revision operator that satisfies vacuity. We intend to prove that $\circledast$ satisfies vacuity. Consider that $A \not \neg \neg$. By expansive credibility $\neg \neg \alpha \in C$. Thus by closure under double negation $\alpha \in C$. Hence $A \circledast \alpha=$ $A * \alpha$. Therefore, it follows by $*$ vacuity that $A \cup\{\alpha\} \subseteq A \circledast \alpha$.

(f) Let $A \nvdash \perp$ and let $C$ be a set that satisfies expansive credibility and single sentence closure. We intend to prove that $\circledast$ satisfies strict improvement. Let $\alpha \in A \circledast \alpha$ and $\vdash \alpha \rightarrow \beta$. Suppose that $\alpha \notin C$. By single sentence closure $\neg \neg \alpha \notin C$. Thus by expansive credibility $A \vdash \neg \alpha$. On the other hand, by definition of $\circledast$, it follows that $A \circledast \alpha=A$. Hence $A \vdash \perp$. Contradiction. Hence $\alpha \in C$. Thus by single sentence closure $\beta \in C$. Therefore $A \circledast \beta=A * \beta$, from which it follows by $*$ success that $\beta \in A \circledast \beta$.

(g) Let $A \nvdash \perp$. We start by noticing that, according to Observation 4.5, if $C$ satisfies either credibility of logical equivalents or uniform credibility or single sentence closure, then it also satisfies closure under double negation. Let $C$ be a set that satisfies expansive credibility, closure under double negation and disjunctive completeness. We intend to prove that $\circledast$ satisfies disjunctive distribution. Let $\alpha \notin A \circledast \alpha$ and $\beta \notin A \circledast \beta$. Thus, by $*$ success, it follows that $\alpha \notin C$ and $\beta \notin C$. By closure under double negation it follows that $\neg \neg \alpha \notin C$ and $\neg \neg \beta \notin C$, from which it follows, by expansive credibility that $A \vdash \neg(\alpha \vee \beta)$. On the other hand, by disjunctive completeness $\alpha \vee \beta \notin C$. Therefore $A \circledast(\alpha \vee \beta)=A$, thus $\alpha \vee \beta \notin A \circledast(\alpha \vee \beta)$ (since $A \forall \perp)$.

(h) Let $C$ and $*$ satisfy condition $(\mathbf{C}-*)$ and assume $C$ satisfies element consistency.

Let $A \cap A \circledast \beta \vdash \neg \beta$ and $\alpha \in \mathcal{L}$. It holds that $A \vdash \neg \beta$ and $A \circledast \beta \vdash \neg \beta$. If $\beta \in C$, then by element consistency $\beta \forall \perp$. On the other hand, $A \circledast \beta=A * \beta$, from which it follows, by $*$ success, that $A * \beta \vdash \perp$ (since it holds that $A \circledast \beta \vdash \neg \beta$ ). This contradicts $*$ consistency. Hence $\beta \notin C$. If $\alpha \in C$, then $A \circledast \alpha=A * \alpha$. On the other hand, by condition $(\mathbf{C}-*)$, it follows that $A \cap A * \alpha \vdash \neg \beta$. Therefore $A \cap A \circledast \alpha \vdash \neg \beta$. If $\alpha \notin C$, then $A \circledast \alpha=A$. Hence $A \cap A \circledast \alpha \vdash \neg \beta$.

Assume that $*$ is a revision operator that satisfies weak extensionality. We intend to prove that $\circledast$ satisfies weak extensionality. Let $\vdash \alpha \leftrightarrow \beta$. Assume that $\alpha \notin C$. If $\beta \in C$, then by element consistency $\beta \forall \perp$. On the other hand, $A \circledast \beta=A * \beta$, from which it follows, by $*$ success and consistency, that $A \circledast \beta \forall \neg \beta$. From $\alpha \notin C$ it follows, by condition $(\mathbf{C}-*)$, that $A \cap A \circledast \beta \vdash \neg \alpha$. Therefore $A \circledast \beta \vdash \neg \alpha$ from which it follows that $A \circledast \beta \vdash \neg \beta$. Contradiction. Hence $\beta \notin C$. By symmetry of the case it also follows that if $\beta \notin C$, then $\alpha \notin C$. Therefore $\alpha \in C$ if and only if $\beta \in C$. Let $\alpha \in C$. Then $\beta \in C$. Hence $A \circledast \alpha=A * \alpha$ and $A \circledast \beta=A * \beta$. From which it follows, by $*$ weak extensionality, that $A \cap A \circledast \alpha=A \cap A \circledast \beta$.

Let $\alpha \notin C$. Then $\beta \notin C$. Hence $A \circledast \alpha=A \circledast \beta=A$. Therefore $A \cap A \circledast \alpha=A \cap A \circledast \beta=A$. 
Assume that $*$ is a revision operator that satisfies uniformity. We intend to prove that $\circledast$ satisfies uniformity. Let it be the case that for all subsets $A^{\prime} \subseteq A, A^{\prime} \cup\{\alpha\} \vdash \perp$ if and only if $A^{\prime} \cup\{\beta\} \vdash \perp$. If $\alpha \in C$ and $\beta \in C$, then $A \circledast \alpha=A * \alpha$ and $A \circledast \beta=A * \beta$. By $*$ uniformity $A \cap A * \alpha=A \cap A * \beta$. Hence $A \cap A \circledast \alpha=A \cap A \circledast \beta$.

If $\alpha \notin C$ and $\beta \notin C$, then $A \circledast \alpha=A \circledast \beta=A$. Hence $A \cap A \circledast \alpha=A \cap A \circledast \beta$.

Assume now by reductio ad absurdum, without loss of generality, that $\alpha \in C$ and $\beta \notin C$. Hence by condition $(\mathbf{C}-*)$ it follows that $A \cap A * \alpha \vdash \neg \beta$. On the other hand, from $\alpha \in C$, it follows that $A \circledast \alpha=A * \alpha$ and, by element consistency, that $\alpha \forall \perp$. Hence, by $*$ consistency $A \circledast \alpha \forall \perp$. By $*$ success it follows that $A \circledast \alpha \forall \neg \alpha$. Thus $A \cap A \circledast \alpha \forall \neg \alpha$. By hypothesis it follows that $A \cap A \circledast \alpha \forall \neg \beta$. Contradiction.

(i) Let $C$ and $*$ satisfy condition $(\mathbf{C}-*)$ and assume $C$ satisfies expansive credibility. Let * be a revision operator that satisfies vacuity. Consider that $A \forall \neg \alpha$. It follows by expansive credibility that $\neg \neg \alpha \in C$. Assume by reductio ad absurdum that $\alpha \notin C$. Hence by condition $(\mathbf{C}-*)$ it follows that $A \cap A * \neg \neg \alpha \vdash \neg \alpha$. From which it follows that $A \vdash \neg \alpha$. Contradiction. Hence $\alpha \in C$. Therefore $A \circledast \alpha=A * \alpha$. Thus, by $*$ vacuity it follows that $A \cup\{\alpha\} \subseteq A \circledast \alpha$.

\section{Proof of Theorem 4.9.}

Let $A$ be a consistent belief base and $\circledast$ be an operator of credibility-limited revision induced by a revision operator $*$ for $A$ and a set $C \subseteq \mathcal{L}$ that satisfies expansive credibility and closure under double negation. We will prove by double inclusion that $C=\{\alpha: \alpha \in A \circledast \alpha\}$. Let $\alpha \in C$. Then $A \circledast \alpha=A * \alpha$, from which it follows by $*$ success that $\alpha \in A \circledast \alpha$. Therefore $C \subseteq\{\alpha: \alpha \in A \circledast \alpha\}$.

Let $\alpha \in A \circledast \alpha$. If $A \circledast \alpha \neq A$, then by definition of $\circledast$ it follows that $\alpha \in C$. Assume now that $A \circledast \alpha=A$. Thus $A \nvdash \neg \alpha$ (since $A \nvdash \perp$ ). By expansive credibility it follows that $\neg \neg \alpha \in C$. Therefore $\alpha \in C$ (since $C$ satisfies closure under double negation). Hence $\{\alpha: \alpha \in A \circledast \alpha\} \subseteq C$.

\section{Proof of Observation 4.10.}

Let $\circledast$ be an operator on $A$ that satisfies consistency preservation. We will show that $C$ satisfies element consistency. Let $\alpha \in C$. Then $\alpha \in A \circledast \alpha$. On the other hand from $A \nvdash \perp$ it follows by $\circledast$ consistency preservation that $A \circledast \alpha \nvdash \perp$. Hence $\alpha \forall \perp$.

Let $\circledast$ be an operator on $A$ that satisfies strict improvement. We will show that $C$ satisfies single sentence closure. Let $\alpha \in C$ and $\beta \in C n(\alpha)$. Hence $\alpha \in A \circledast \alpha$ and $\vdash \alpha \rightarrow \beta$. Thus, by strict improvement, $\beta \in A \circledast \beta$. Therefore $\beta \in C$.

Let $\circledast$ be an operator on $A$ that satisfies disjunctive distribution. We will show that $C$ satisfies disjunctive completeness. Let $\alpha \vee \beta \in C$. Hence $\alpha \vee \beta \in A \circledast(\alpha \vee \beta)$. Thus, by disjunctive distribution, $\alpha \in A \circledast \alpha$ or $\beta \in A \circledast \beta$. From which it follows that $\alpha \in C$ or $\beta \in C$.

Let $\circledast$ be an operator on $A$ that satisfies vacuity. We will show that $C$ satisfies expansive credibility and credibility lower bounding.

Expansive credibility: Let $A \nvdash \alpha$. Then $A \nvdash \neg \neg \alpha$. By $\circledast$ vacuity it follows that 
$\neg \alpha \in A \circledast \neg \alpha$. Therefore $\neg \alpha \in C$.

Credibility lower bounding: Let $A \vdash \alpha$. It follows that $A \nvdash \neg \alpha$, since $A \nvdash \perp$. Thus, by $\circledast$ vacuity, $\alpha \in A \circledast \alpha$. Hence $\alpha \in C$.

Let $\circledast$ be an operator on $A$ that satisfies consistency preservation, persistence, relative success and vacuity. By Observation 4.7 it follows that $\circledast$ also satisfies disjunctive distribution, strict improvement and regularity. Thus as shown above $C$ satisfies single sentence closure and disjunctive completeness. Since $C$ satisfies single sentence closure then it also satisfies credibility of logical equivalents. We will now show that $C$ also satisfies revision credibility.

Revision credibility Let $\alpha \in C$ and $\beta \in C n(A \circledast \alpha)$. Hence, by $\circledast$ regularity, it follows that $\beta \in A \circledast \beta$. Hence $\beta \in C$, by definition of $C$. We will now show that $C$ satisfies uniform credibility.

Uniform credibility Assume that it holds for all subsets $A^{\prime}$ of $A$ that $A^{\prime} \cup\{\alpha\} \vdash \perp$ if and only if $A^{\prime} \cup\{\beta\} \vdash \perp$. We intend to prove that $\alpha \in C$ holds if and only if $\beta \in C$ holds. Consider that $\beta \notin C$. Hence $\beta \notin A \circledast \beta$. By $\circledast$ relative success and vacuity it follows, respectively, that $A \circledast \beta=A$ and $A \vdash \neg \beta$. Hence $A \cap A \circledast \beta \vdash \neg \beta$. By persistence it follows that $A \cap A \circledast \alpha \vdash \neg \beta$. From which it follows by hypothesis that $A \cap A \circledast \alpha \vdash \neg \alpha$. Hence $A \circledast \alpha \vdash \neg \alpha$. By consistency preservation it follows that $\alpha \notin A \circledast \alpha$. Thus $\alpha \notin C$. By symmetry of the case it follows that if $\alpha \notin C$, then $\beta \notin C$.

Let $\circledast$ be an operator on $A$ that satisfies relative success, uniformity, vacuity and consistency preservation. We will show that $C$ satisfies uniform credibility. Assume that it holds for all subsets $A^{\prime}$ of $A$ that $A^{\prime} \cup\{\alpha\} \vdash \perp$ if and only if $A^{\prime} \cup\{\beta\} \vdash \perp$. Hence, by $\circledast$ uniformity, $A \cap A \circledast \alpha=A \cap A \circledast \beta$. Let $\alpha \notin C$. Thus $\alpha \notin A \circledast \alpha$. Therefore by $\circledast$ vacuity $A \vdash \neg \alpha$ and by $\circledast$ relative success $A \circledast \alpha=A$. Thus $A=A \cap A \circledast \beta$. Therefore $A \cap A \circledast \beta \vdash \neg \alpha$. From which it follows, by hypothesis, that $A \cap A \circledast \beta \vdash \neg \beta$. Thus $A \circledast \beta \vdash \neg \beta$. By $\circledast$ consistency preservation it follows that $\beta \notin A \circledast \beta$. Hence $\beta \notin C$. By symmetry of the case it follows from $\beta \notin C$ that $\alpha \notin C$. Therefore $\alpha \in C$ if and only if $\beta \in C$.

Let $\circledast$ be an operator on $A$ that satisfies relative success, vacuity, consistency preservation and weak extensionality. ${ }^{22}$ We will show that $C$ satisfies credibility of logical equivalents. Let $\vdash \alpha \leftrightarrow \beta$. Suppose that $\alpha \notin C$. Hence $\alpha \notin A \circledast \alpha$. Thus by relative success $A \circledast \alpha=A$. Assume by reductio ad absurdum that $\beta \in C$. Thus $\beta \in A \circledast \beta$. Thus, by $\circledast$ consistency preservation, $A \circledast \beta \not \neg \neg$. By weak extensionality it follows that $A \cap A \circledast \alpha=A \cap A \circledast \beta$. Thus $A \cap A \circledast \alpha \forall \neg \beta$, from which it follows that $A \cap A \circledast \alpha \forall \neg \alpha$. Therefore, $A \forall \neg \alpha$. From which it follows by vacuity that $\alpha \in A \circledast \alpha$. Contradiction. Hence if $\alpha \notin C$, then $\beta \notin C$. By symmetry of the case it follows that $\beta \notin C$, then $\alpha \notin C$. Therefore $\alpha \in C$ if and only if $\beta \in C$.

22. Note that according to Observation 3.5 if $\circledast$ satisfies uniformity, then it also satisfies weak extensionality. 


\section{Proof of Observation 4.11.}

Let $A$ be a consistent belief base, $\circledast$ be an operator on $A$ and $C=\{\alpha: \alpha \in A \circledast \alpha\}$. Let $*$ be the operator on $A$ defined by:

$$
A * \alpha=\left\{\begin{array}{ll}
A \circledast \alpha & \text { if } \alpha \in C \\
(A \backslash \bigcup(A \Perp \neg \alpha)) \cup\{\alpha\} & \text { otherwise }
\end{array} .\right.
$$

In what follows we show that this operator satisfies statements (a), (b) and (c).

(a) We start by showing that the following equality holds:

$$
A \circledast \alpha= \begin{cases}A * \alpha & \text { if } \alpha \in C \\ A & \text { otherwise }\end{cases}
$$

If $\alpha \in C$, then by definition of $*, A \circledast \alpha=A * \alpha$. Assume now that $\alpha \notin C$. Hence $\alpha \notin A \circledast \alpha$, from which it follows, by $\circledast$ relative success, that $A \circledast \alpha=A$.

It remains to show that $*$ is a revision operator. According to Definition 2.3 we must to show that $*$ satisfies success, inclusion and consistency.

That $*$ satisfies success follows trivially by $*$ definition.

That $*$ satisfies inclusion follows trivially by $*$ definition and $\circledast$ inclusion.

Let $\alpha \nvdash \perp$. If $\alpha \in C$, then $A * \alpha=A \circledast \alpha$. Thus, by $\circledast$ consistency preservation, it follows that $A * \alpha \nvdash \perp$. Assume now that $\alpha \notin C$. Thus, by Lemma 4, it follows that $A * \alpha \nvdash \perp$.

(b) Assume $\circledast$ satisfies vacuity. We will prove that $*$ satisfies vacuity. If $\alpha \in C$, then $A * \alpha=A \circledast \alpha$ and the rest of the proof for this case follows by $\circledast$ vacuity. If $\alpha \notin C$, then $A * \alpha=(A \backslash \bigcup(A \Perp \neg \alpha)) \cup\{\alpha\}$. The rest of the proof follows trivially by Lemma 4 .

Assume $\circledast$ satisfies relevance. We will prove that $*$ satisfies relevance. If $\alpha \in C$, then $A * \alpha=A \circledast \alpha$ and the rest of the proof for this case follows by $\circledast$ relevance. Let $\alpha \notin C$. Hence $A * \alpha=(A \backslash \bigcup(A \Perp \neg \alpha)) \cup\{\alpha\}$. The rest of the proof follows trivially by Lemma 4 .

Assume $\circledast$ satisfies core-retainment. We will prove that $*$ satisfies core-retainment. If $\alpha \in C$, then $A * \alpha=A \circledast \alpha$ and the rest of the proof for this case follows by $\circledast$ core-retainment. Let $\alpha \notin C$. Hence $A * \alpha=(A \backslash \bigcup(A \Perp \neg \alpha)) \cup\{\alpha\}$. The rest of the proof follows trivially by Lemma 4.

Assume $\circledast$ satisfies disjunctive elimination. We will prove that $*$ satisfies disjunctive elimination. If $\alpha \in C$, then $A * \alpha=A \circledast \alpha$ and the rest of the proof for this case follows by $\circledast$ disjunctive elimination. Let $\alpha \notin C$. Hence $A * \alpha=(A \backslash \bigcup(A \Perp \neg \alpha)) \cup\{\alpha\}$. The rest of the proof follows trivially by Lemma 4 .

Assume $\circledast$ satisfies uniformity, relative success, vacuity and consistency preservation. We will prove that $*$ satisfies uniformity. Assume that it holds for all subsets $A^{\prime} \subseteq$ $A, A^{\prime} \cup\{\alpha\} \vdash \perp$ if and only if $A^{\prime} \cup\{\beta\} \vdash \perp$. Let $\beta \notin C$. Hence $\beta \notin A \circledast \beta$. Thus, by $\circledast$ relative success it follows that $A \circledast \beta=A$ and by vacuity that $A \vdash \neg \beta$. Hence $A \cap A \circledast \beta \vdash \neg \beta$. Therefore, by hypothesis $A \cap A \circledast \beta \vdash \neg \alpha$. Thus by $\circledast$ uniformity $A \cap A \circledast \alpha \vdash \neg \alpha$. From which it follows that $A \circledast \alpha \vdash \neg \alpha$. Hence, by $\circledast$ consistency 
preservation, it follows that $\alpha \notin C$. By symmetry of the case it follows that if $\alpha \notin C$, then $\beta \notin C$. Therefore $\alpha \in C$ if and only if $\beta \in C$.

Let $\alpha \in C$, then $\beta \in C$. Hence $A * \alpha=A \circledast \alpha$ and $A * \beta=A \circledast \beta$. Thus by $\circledast$ uniformity $A \cap A * \alpha=A \cap A * \beta$.

Let $\alpha \notin C$, then $\beta \notin C$. Then $A * \alpha=(A \backslash \bigcup(A \Perp \neg \alpha)) \cup\{\alpha\}$ and $A * \beta=(A \backslash$ $\bigcup(A \Perp \neg \beta)) \cup\{\beta\}$. From which it follows by Lemma 4 that $A \cap A * \alpha=A \cap A * \beta$.

Assume $\circledast$ satisfies weak extensionality, relative success, vacuity and consistency preservation. We will prove that $*$ satisfies weak extensionality. Let $\alpha$ and $\beta$ be such that $\vdash \alpha \leftrightarrow \beta$. Let $\beta \notin C$. Hence, by $\circledast$ relative success it follows that $A \circledast \beta=A$ and by vacuity that $A \vdash \neg \beta$. Hence $A \cap A \circledast \beta \vdash \neg \beta$. Therefore, by hypothesis $A \cap A \circledast \beta \vdash \neg \alpha$. Thus by $\circledast$ weak extensionality $A \cap A \circledast \alpha \vdash \neg \alpha$. From which it follows that $A \circledast \alpha \vdash \neg \alpha$. Hence, by $\circledast$ consistency preservation, it follows that $\alpha \notin C$. By symmetry of the case it follows that if $\alpha \notin C$, then $\beta \notin C$. Therefore $\alpha \in C$ if and only if $\beta \in C$.

Let $\alpha \in C$, then $\beta \in C$. Hence $A * \alpha=A \circledast \alpha$ and $A * \beta=A \circledast \beta$. Thus by $\circledast$ weak extensionality $A \cap A * \alpha=A \cap A * \beta$.

Let $\alpha \notin C$, then $\beta \notin C$. Then $A * \alpha=(A \backslash \bigcup(A \Perp \neg \alpha)) \cup\{\alpha\}$ and $A * \beta=$ $(A \backslash \bigcup(A \Perp \neg \beta)) \cup\{\beta\}$. From which it follows by Lemma 4 that $A \cap A * \alpha=A \cap A * \beta$.

Assume $\circledast$ satisfies weak relative closure. We will prove that $*$ satisfies weak relative closure. If $\alpha \in C$, then $A * \alpha=A \circledast \alpha$ and the rest of the proof for this case follows by $\circledast$ weak relative closure. Let $\alpha \notin C$. Hence $A * \alpha=(A \backslash \bigcup(A \Perp \neg \alpha)) \cup\{\alpha\}$. The rest of the proof follows trivially by Lemma 4 .

(c) Consider that $\circledast$ satisfies persistence, relative success and vacuity. We intend to prove that $C$ and $*$ satisfy condition $(\mathbf{C}-*)$.

Let $\alpha \notin C$ and $\beta \in C$. Then, by relative success, $A \circledast \alpha=A$. By definition of $*$ it follows that $A * \beta=A \circledast \beta$.

If $A \nvdash \neg \alpha$, then by $\circledast$ vacuity it follows that $\alpha \in A \circledast \alpha$. From which it follows that $\alpha \in C$. Contradiction.

Hence $A \vdash \neg \alpha$. Therefore $A \cap A \circledast \alpha \vdash \neg \alpha$. Hence, by $\circledast$ persistence, $A \cap A \circledast \beta \vdash \neg \alpha$. Thus $A \cap A * \beta \vdash \neg \alpha$.

\section{Proof of Theorem 5.2.}

Let $A$ be a consistent belief base.

$((\mathrm{a}) \rightarrow(\mathrm{b}))$ Let $\circledast$ be an operator on $A$ that satisfies relative success, consistency preservation and inclusion. Let $C$ be the set defined by:

$$
C=\{\alpha: \alpha \in A \circledast \alpha\}
$$

According to Observation 4.10, $C$ satisfies element consistency. On the other hand, it follows from Observation 4.11 (a) that there exists an operator $*$ such that $\circledast$ is the credibility-limited base revision induced by $*$ and $C$.

$((\mathrm{b}) \rightarrow(\mathrm{a}))$ Let $\circledast$ be the operator of credibility-limited base revision induced by a revision operator $*$ and a set $C \subseteq \mathcal{L}$ that satisfies element consistency. Hence by Observation 4.8 
(a) and (b), $\circledast$ satisfies relative success, consistency preservation and inclusion.

Proofs of Theorems 5.4, 5.7, 5.9.

(Right-to-left)

Let $A$ be a consistent belief base and $\circledast$ be an operator that satisfies relative success, consistency preservation, inclusion, vacuity and uniformity. Let $C$ be the set defined by:

$$
C=\{\alpha: \alpha \in A \circledast \alpha\}
$$

It follows from Observation 4.10 that $C$ satisfies element consistency, expansive credibility and uniform credibility.

Furthermore, from Observation 4.10, it follows that:

- If $\circledast$ satisfies strict improvement, then $C$ satisfies single sentence closure;

- If $\circledast$ satisfies disjunctive distribution, then $C$ satisfies disjunctive completeness;

From Observation 4.11 (a) it follows that there exists a revision operator $*$ such that $\circledast$ is the credibility-limited base revision induced by $*$ and $C$. Furthermore from Observation 4.11 (c) it follows that if $\circledast$ satisfies persistence, then $C$ and $*$ satisfy condition $(\mathbf{C}-*)$.

For Theorem 5.4

From Observation 4.11 and Observation 2.7 it follows that $*$ is a partial meet revision.

For Theorem 5.7

From Observation 4.11 and Observation 2.11 it follows that $*$ is a kernel revision.

For Theorem 5.9

From Observation 4.11 and Observation 3.2 it follows that $*$ is a smooth kernel revision. (Left-to-right)

Let $A$ be a consistent belief base, $*$ be an operator on $A$ and $C \subseteq \mathcal{L}$. Let $\circledast$ be such that:

$$
A \circledast \alpha= \begin{cases}A * \alpha & \text { if } \alpha \in C \\ A & \text { otherwise }\end{cases}
$$

From Observation 4.8 it follows that if $*$ satisfies success, consistency, inclusion, vacuity and uniformity, then:

- $\circledast$ satisfies inclusion and relative success.

- If $C$ satisfies element consistency, then $\circledast$ satisfies consistency preservation.

- If $C$ satisfies uniform credibility, then $\circledast$ satisfies uniformity.

- If $C$ satisfies expansive credibility and uniform credibility, then $\circledast$ satisfies vacuity.

- If $C$ satisfies expansive credibility and single sentence closure, then $\circledast$ satisfies strict improvement.

- If $C$ satisfies expansive credibility, uniform credibility and disjunctive completeness, then $\circledast$ satisfies disjunctive distribution.

- If $C$ satisfies expansive credibility and $C$ and $*$ satisfy condition $(\mathbf{C}-*)$, then $\circledast$ satisfies vacuity.

- If $C$ satisfies element consistency and $C$ and $*$ satisfy condition $(\mathbf{C}-*)$, then $\circledast$ satisfies persistence and uniformity.

For Theorem 5.4

If $*$ is a partial meet revision operator on $A$, then from Observation $2.7 *$ satisfies success, consistency, inclusion, relevance and uniformity. From Observation 3.5 it follows that * 
satisfies vacuity. From Observation 4.8 (a) it holds also that $\circledast$ satisfies relevance.

\section{For Theorem 5.7}

If $*$ is a kernel revision operator on $A$, then from Observation $2.11 *$ satisfies success, consistency, inclusion, core-retainment and uniformity. From Observation 3.5 it follows that * satisfies vacuity. From Observation 4.8 (a) it holds also that $\circledast$ satisfies core-retainment.

\section{For Theorem 5.9}

If $*$ is a smooth kernel revision operator on $A$, then $*$ is a kernel revision operator and $*$ satisfies weak relative closure (Observation 3.2). From Observation 3.5 it follows that * satisfies vacuity. From Observation 4.8 (a) it holds also that $\circledast$ satisfies core-retainment and weak relative closure.

\section{Proof of Theorem 5.11.}

\section{(Right-to-left)}

Let $A$ be a consistent belief base and $\circledast$ be an operator that satisfies relative success, consistency preservation, inclusion, vacuity, weak extensionality and disjunctive elimination. Let $C$ be the set defined by:

$$
C=\{\alpha: \alpha \in A \circledast \alpha\}
$$

It follows from Observation 4.10 that $C$ satisfies element consistency, expansive credibility and credibility of logical equivalents.

Furthermore, from Observation 4.10, it follows that:

- If $\circledast$ satisfies strict improvement, then $C$ satisfies single sentence closure;

- If $\circledast$ satisfies disjunctive distribution, then $C$ satisfies disjunctive completeness;

From Observations 3.4 and 4.11 it follows that there exists a basic AGM-generated base revision $*$ such that $\circledast$ is the credibility-limited base revision induced by $*$ and $C$. Furthermore from Observation 4.11 (c) it follows that if $\circledast$ satisfies persistence, then $C$ and $*$ satisfy condition $(\mathbf{C}-*)$.

(Left-to-right)

Let $A$ be a consistent belief base, $C \subseteq \mathcal{L}$ and $*$ be a basic AGM-generated revision operator on $A$. Let $\circledast$ be such that:

$$
A \circledast \alpha= \begin{cases}A * \alpha & \text { if } \alpha \in C \\ A & \text { otherwise }\end{cases}
$$

From Observation $3.4 *$ satisfies success, consistency, inclusion, vacuity, weak extensionality and disjunctive elimination. Hence from Observation 4.8 (a) it follows that $\circledast$ satisfies relative success, inclusion and disjunctive elimination. Furthermore, it follows from Observation 4.8 that:

- If $C$ satisfies element consistency, then $\circledast$ satisfies consistency preservation.

- If $C$ satisfies credibility of logical equivalents, then $\circledast$ satisfies weak extensionality.

- If $C$ satisfies expansive credibility and credibility of logical equivalents, then $\circledast$ satisfies vacuity.

- If $C$ satisfies expansive credibility and single sentence closure, then $\circledast$ satisfies strict improvement.

- If $C$ satisfies single sentence closure, then $\circledast$ satisfies weak extensionality.

- If $C$ satisfies expansive credibility and single sentence closure, then $\circledast$ satisfies vacuity. 
- If $C$ satisfies expansive credibility, credibility of logical equivalents and disjunctive completeness, then $\circledast$ satisfies disjunctive distribution.

- If $C$ satisfies expansive credibility, single sentence closure and disjunctive completeness, then $\circledast$ satisfies disjunctive distribution.

- If $C$ satisfies expansive credibility and $C$ and $*$ satisfy condition $(\mathbf{C}-*)$, then $\circledast$ satisfies vacuity.

- If $C$ satisfies element consistency and $C$ and $*$ satisfy condition $(\mathbf{C}-*)$, then $\circledast$ satisfies persistence and weak extensionality.

Proof of Observation 6.1.

Proof that P-CLPMR $\subset$ SI+DD-CLPMR, SI+DD-CLPMR $\subset$ DD-CLPMR, SI+DD$\mathrm{CLPMR} \subset$ SI-CLPMR, DD-CLPMR $\nsubseteq$ SI-CLPMR, SI-CLPMR $\nsubseteq$ DD-CLPMR, DD$\mathrm{CLPMR} \subset \mathrm{CLPMR}$ and SI-CLPMR $\subset \mathrm{CLPMR}:$

It follows from Example 5.5 that:

(i) CLPMR $\nsubseteq$ DD-CLPMR, SI-CLPMR $\nsubseteq$ DD-CLPMR and SI-CLPMR $\nsubseteq$ SI+DDCLPMR;

(ii) CLPMR $\nsubseteq$ SI-CLPMR, DD-CLPMR $\nsubseteq$ SI-CLPMR and DD-CLPMR $\nsubseteq$ SI+DDCLPMR;

(iii) SI+DD-CLPMR $\nsubseteq$ P-CLPMR.

On the other hand it follows from Theorem 5.4 that SI+DD-CLPMR $\subseteq$ DD-CLPMR, $\mathrm{SI}+\mathrm{DD}-\mathrm{CLPMR} \subseteq \mathrm{SI}-\mathrm{CLPMR}, \mathrm{DD}-\mathrm{CLPMR} \subseteq \mathrm{CLPMR}$ and SI-CLPMR $\subseteq \mathrm{CLPMR}$. Furthermore, combining Theorem 5.4 and Observation 4.7 we can conclude that P-CLPMR $\subseteq \mathrm{SI}+\mathrm{DD}-\mathrm{CLPMR}$.

Proof that $\mathrm{P}-\mathrm{CLKR} \subset \mathrm{SI}+\mathrm{DD}-\mathrm{CLKR}, \mathrm{SI}+\mathrm{DD}-\mathrm{CLKR} \subset \mathrm{DD}-\mathrm{CLKR}, \mathrm{SI}+\mathrm{DD}-\mathrm{CLKR}$ $\subset$ SI-CLKR, DD-CLKR $\nsubseteq$ SI-CLKR, SI-CLKR $\nsubseteq \subset$ DD-CLKR, DD-CLKR $\subset$ CLKR and SI-CLKR $\subset$ CLKR:

That $\mathrm{P}-\mathrm{CLKR} \subseteq \mathrm{SI}+\mathrm{DD}-\mathrm{CLKR}$ follows trivially from Theorem 5.7 and Observation 4.7. That $\mathrm{SI}+\mathrm{DD}-\mathrm{CLKR} \subseteq \mathrm{DD}-\mathrm{CLKR}, \mathrm{SI}+\mathrm{DD}-\mathrm{CLKR} \subseteq \mathrm{SI}-\mathrm{CLKR}, \mathrm{DD}-\mathrm{CLKR} \subseteq \mathrm{CLKR}$ and SI-CLKR $\subseteq$ CLKR follow trivially from Theorem 5.7.

To prove that SI+DD-CLKR $\nsubseteq$ P-CLKR, DD-CLKR $\nsubseteq$ SI+DD-CLKR, SI-CLKR $\nsubseteq$ SI+DD-CLKR, SI-CLKR $\nsubseteq$ DD-CLKR, DD-CLKR $\nsubseteq$ SI-CLKR, CLKR $\nsubseteq$ DD-CLKR and $\mathrm{CLKR} \nsubseteq \mathrm{SI}-\mathrm{CLKR}$ it is enough to consider the credibility-limited revisions presented in Example 5.5, attending to Definition 5.6, Theorem 5.7 and to the fact that every partial meet revision is a kernel revision (Observation 3.6).

Proof that P-CLSKR $\subset \mathrm{SI}+\mathrm{DD}$-CLSKR, SI+DD-CLSKR $\subset$ DD-CLSKR, SI+DDCLSKR $\subset$ SI-CLSKR, DD-CLSKR $\nsubseteq$ SI-CLSKR, SI-CLSKR $\nsubseteq$ DD-CLSKR, DD-CLSKR $\subset$ CLSKR and SI-CLSKR $\subset$ CLSKR:

That P-CLSKR $\subseteq$ SI+DD-CLSKR follows trivially from Theorem 5.9 and Observation 4.7. That $\mathrm{SI}+\mathrm{DD}-\mathrm{CLSKR} \subseteq \mathrm{DD}-\mathrm{CLSKR}, \mathrm{SI}+\mathrm{DD}-\mathrm{CLSKR} \subseteq \mathrm{SI}-\mathrm{CLSKR}, \mathrm{DD}-\mathrm{CLSKR} \subseteq$ CLSKR and SI-CLSKR $\subseteq$ CLSKR follow trivially from Theorem 5.9.

To prove that SI+DD-CLSKR $\nsubseteq$ P-CLSKR, DD-CLSKR $\nsubseteq$ SI+DD-CLSKR, SI-CLSKR 
$\nsubseteq \mathrm{SI}+\mathrm{DD}$-CLSKR, SI-CLSKR $\nsubseteq$ DD-CLSKR, DD-CLSKR $\nsubseteq$ SI-CLSKR, CLSKR $\nsubseteq$ DD-CLSKR and CLSKR $\nsubseteq$ SI-CLSKR it is enough to consider the credibility-limited revisions presented in Example 5.5, attending to Definition 5.8, Theorem 5.9 and to the fact that every partial meet revision is a smooth kernel revision (Observation 3.6).

Proof that P-CLbAGMR $\subset$ SI+DD-CLbAGMR, SI+DD-CLbAGMR $\subset$ DDCLbAGMR, SI+DD-CLbAGMR $\subset$ SI-CLbAGMR, DD-CLbAGMR $\nsubseteq$ SI-CLbAGMR, SI-CLbAGMR $\nsubseteq$ DD-CLbAGMR, DD-CLbAGMR $\subset$ CLbAGMR and SI-CLbAGMR $\subset$ CLbAGMR:

That P-CLbAGMR $\subseteq$ SI+DD-CLbAGMR follows trivially from Theorem 5.11 and Observation 4.7.

That SI+DD-CLbAGMR $\subseteq$ DD-CLbAGMR, SI+DD-CLbAGMR $\subseteq$ SI-CLbAGMR, $\mathrm{DD}-\mathrm{CLbAGMR} \subseteq \mathrm{CLbAGMR}$ and SI-CLbAGMR $\subseteq$ CLbAGMR follow trivially from Theorem 5.11.

To prove that SI+DD-CLbAGMR $\nsubseteq$ P-CLbAGMR, DD-CLbAGMR $\nsubseteq$ SI+DD-CLbAGMR, SI-CLbAGMR $\nsubseteq$ SI+DD-CLbAGMR, SI-CLbAGMR $\nsubseteq$ DD-CLbAGMR, DD-CLbAGMR $\nsubseteq$ SI-CLbAGMR, CLbAGMR $\nsubseteq$ DD-CLbAGMR and CLbAGMR $\nsubseteq$ SI-CLbAGMR it is enough to consider the credibility-limited revisions presented in Example 5.5, attending to Definition 5.10, Theorem 5.11 and to the fact that every partial meet revision is a basic AGM-generated base revision (Observation 3.6).

\section{Proof of Observation 6.3.}

It follows trivially from Observation 3.6 that CLPMR $\subseteq$ CLSKR $\subseteq$ CLKR, SI-CLPMR $\subseteq \mathrm{SI}-\mathrm{CLSKR} \subseteq \mathrm{SI}-\mathrm{CLKR}, \mathrm{DD}-\mathrm{CLPMR} \subseteq \mathrm{DD}-\mathrm{CLSKR} \subseteq \mathrm{DD}-\mathrm{CLKR}, \mathrm{SI}+\mathrm{DD}-\mathrm{CLPMR} \subseteq$ $\mathrm{SI}+\mathrm{DD}-\mathrm{CLSKR} \subseteq \mathrm{SI}+\mathrm{DD}-\mathrm{CLKR}$ and $\mathrm{P}-\mathrm{CLPMR} \subseteq \mathrm{P}-\mathrm{CLSKR} \subseteq \mathrm{P}-\mathrm{CLKR}$.

That CLSKR $\nsubseteq$ CLPMR, SI-CLSKR $\nsubseteq$ SI-CLPMR, DD-CLSKR $\nsubseteq$ DD-CLPMR, SI+DD-CLSKR $\nsubseteq \subseteq$ SI+DD-CLPMR and P-CLSKR $\nsubseteq$ P-CLPMR, follows from Observation 6.1 and Example 6.2 (a) (see Footnote 20).

That CLKR $\nsubseteq$ CLSKR, SI-CLKR $\nsubseteq$ SI-CLSKR, DD-CLKR $\nsubseteq$ DD-CLSKR, SI+DDCLKR $\nsubseteq \mathrm{SI}+\mathrm{DD}$-CLSKR and P-CLKR $\nsubseteq$ P-CLSKR, follows from Observation 6.1 and Example 6.2 (b) (see Footnote 20).

\section{Proof of Observation 6.5.}

It follows trivially from Observation 3.6 that CLPMR $\subseteq$ CLbAGMR, SI-CLPMR $\subseteq$ SI-CLbAGMR, DD-CLPMR $\subseteq$ DD-CLbAGMR, SI+DD-CLPMR $\subseteq$ SI+DD-CLbAGMR and $\mathrm{P}-\mathrm{CLPMR} \subseteq \mathrm{P}-\mathrm{CLbAGMR}$.

On the other hand, according to Example 6.4, P-CLbAGMR $\nsubseteq$ CLPMR and from this, having in mind Observation 6.1, it follows that CLbAGMR $\nsubseteq$ CLPMR, SI-CLbAGMR $\nsubseteq$ SI-CLPMR, DD-CLbAGMR $\nsubseteq$ DD-CLPMR, SI+DD-CLbAGMR $\nsubseteq$ SI+DD-CLPMR and P-CLbAGMR $\nsubseteq$ P-CLPMR. 


\section{Proof of Observation 6.7.}

According to Examples 6.4 and 6.6 it holds, respectively, that P-CLbAGMR $\nsubseteq$ CLKR and that P-CLSKR $\nsubseteq$ CLbAGMR. Therefore it follows from Observations 6.1 and 6.3 that all the statements of Observation 6.7 hold.

\section{References}

Alchourrón, C., Gärdenfors, P., \& Makinson, D. (1985). On the logic of theory change: Partial meet contraction and revision functions. Journal of Symbolic Logic, 50, 510530 .

Alchourrón, C., \& Makinson, D. (1981). Hierarchies of regulations and their logic. In Hilpinen, R. (Ed.), New Studies in Deontic Logic: Norms, Actions, and the Foundations of Ethics, pp. 125-148.

Alchourrón, C., \& Makinson, D. (1985). On the logic of theory change: Safe contraction. Studia Logica, 44, 405-422.

Booth, R., Fermé, E., Konieczny, S., \& Pino Pérez, R. (2012). Credibility-limited revision operators in propositional logic. In 13th International Conference on the Principles of Knowledge Representation and Reasoning, KR 2012, pp. 116-125.

Booth, R., \& Hunter, A. (2018). Trust as a precursor to belief revision. Journal of Artificial Intelligence Research, 61, 699-722.

Dalal, M. (1988). Investigations into a theory of knowledge base revision: Preliminary report. In Seventh National Conference on Artificial Intelligence, (AAAI-88), pp. 475-479, St. Paul.

Dragoni, A. F., Giorgini, P., \& Baffetti, M. (1997). Distributed belief revision vs. belief revision in a multi-agent environment: First results of a simulation experiment. In European Workshop on Modelling Autonomous Agents in a Multi-Agent World, pp. 45-62. Springer.

Falappa, M. A., Kern-Isberner, G., Reis, M. D. L., \& Simari, G. R. (2012). Prioritized and non-prioritized multiple change on belief bases. Journal of Philosophical Logic, 41(1), $77-113$.

Fermé, E. (1992). Actualización de bases de conocimiento usando teorías de cambio de creencia. In 3rd Ibero-American Conference on Artificial Intelligence 1992, pp. 419436.

Fermé, E., Garapa, M., \& Reis, M. D. L. (2017). On ensconcement and contraction. Journal of Logic and Computation, 27(7), 2011-2042.

Fermé, E., \& Hansson, S. O. (1999). Selective revision. Studia Logica, 63:3, 331-342.

Fermé, E., \& Hansson, S. O. (2001). Shielded contraction. In H.Rott, \& Williams, M.A. (Eds.), Frontiers in Belief Revision, Applied Logic Series, pp. 85-107. Kluwer Academic Publishers.

Fermé, E., \& Hansson, S. O. (2011). AGM 25 years: Twenty-five years of research in belief change. Journal of Philosophical Logic, 40, 295-331. 
Fermé, E., \& Hansson, S. O. (2018.). Belief Change: Introduction and Overview. Springer Briefs in Computer Science Series. Springer.

Fermé, E., Krevneris, M., \& Reis, M. (2008). An axiomatic characterization of ensconcement-based contraction. Journal of Logic and Computation, 18(5), 739-753.

Fermé, E., Mikalef, J., \& Taboada, J. (2003). Credibility-limited functions for belief bases. Journal of Logic and Computation, 13:1, 99-110.

Fuhrmann, A. (1988). Relevant Logic, Modal Logic and Theory Change. Ph.D. thesis, Department of Philosophy and Automated Reasoning Project, Institute of Advanced Studies, Australian National University, Camberra.

Fuhrmann, A. (1991). Theory contraction through base contraction. Journal of Philosophical Logic, 20, 175-203.

Garapa, M. (2017). Advances on belief base dynamics. Ph.D. thesis, Universidade da Madeira.

Garapa, M., Fermé, E., \& Reis, M. (2018a). Studies in credibility-limited base revision. In Proceedings of the Sixteenth International Conference on Principles of Knowledge Representation and Reasoning (KR 2018), pp. 240-247.

Garapa, M., Fermé, E., \& Reis, M. D. (2018b). Shielded base contraction. Artificial Intelligence, 259, 186 - 216.

Gärdenfors, P. (1982). Rules for rational changes of belief. In Pauli, T. (Ed.), Philosophical Essays dedicated to Lennart Aqvist on his fiftieth birthday, No. 34 in Philosophical Studies, pp. 88-101.

Gärdenfors, P. (1988). Knowledge in Flux: Modeling the Dynamics of Epistemic States. The MIT Press, Cambridge.

Gärdenfors, P., \& Rott, H. (1995). Belief revision. In Gabbay, D. M., Hogger, C. J., \& Robinson, J. A. (Eds.), Handbook of Logic in Artificial Intelligence and Logic Programming (Vol. 4), pp. 35-132. Oxford University Press, Oxford, UK.

Hansson, S. O. (1989). New operators for theory change. Theoria, 55, 114-132.

Hansson, S. O. (1991a). Belief Base Dynamics. Ph.D. thesis, Uppsala University.

Hansson, S. O. (1991b). Belief contraction without recovery. Studia Logica, 50, 251-260.

Hansson, S. O. (1992a). A dyadic representation of belief. In Gärdenfors, P. (Ed.), Belief Revision, No. 29 in Cambridge Tracts in Theoretical Computer Science, pp. 89-121. Cambridge University Press.

Hansson, S. O. (1992b). In defense of base contraction. Synthese, 91, 239-245.

Hansson, S. O. (1993). Reversing the Levi identity. Journal of Philosophycal Logic, 22, 637-669.

Hansson, S. O. (1994a). Kernel contraction. Journal of Symbolic Logic, 59, 845-859.

Hansson, S. O. (1994b). Taking belief bases seriously. In Prawitz, \& Westerståhl (Eds.), Logic and Philosophy of Science in Uppsala, pp. 13-28. Kluwer Academic Publishers, Dordrecht. 
Hansson, S. O. (1996). A test battery for rational database updating. Artificial Intelligence, 82, 341-352.

Hansson, S. O. (1999a). A survey of non-prioritized belief revision. Erkenntnis, 50, 413-427.

Hansson, S. O. (1999.b). A Textbook of Belief Dynamics. Theory Change and Database Updating. Applied Logic Series. Kluwer Academic Publishers, Dordrecht.

Hansson, S. O., Fermé, E., Cantwell, J., \& Falappa, M. (2001). Credibility-limited revision. Journal of Symbolic Logic, 66(4), 1581-1596.

Katsuno, H., \& Mendelzon, A. (1992). On the difference between updating a knowledge base and revising it. In Gärdenfors, P. (Ed.), Belief Revision, No. 29 in Cambridge Tracts in Theoretical Computer Science, pp. 183-203. Cambridge University Press.

Liberatore, P. (2018). Belief integration and source reliability assessment. Journal of Artificial Intelligence Research, 63, 87-143.

Makinson, D. (1997). Screened revision. Theoria, 63, 14-23.

Nebel, B. (1989). A knowledge level analysis of belief revision. In Proceedings of the 1st International Conference of Principles of Knowledge Representation and Rea-soning, pp. 301-311. Morgan Kaufmann.

Rott, H. (2000). "Just because". Taking belief bases seriously.. In Buss, S., Hajek, P., \& Pudlak, P. (Eds.), Logic Colloquium '98 - Proceedings of the Annual European Summer Meeting of the Association for Symbolic Logic. Lecture Notes in Logic, Vol. 13, Prague. Association for Symbolic Logic.

Tamargo, L. H., García, A. J., Falappa, M. A., \& Simari, G. R. (2014). On the revision of informant credibility orders. Artificial Intelligence, 212, 36-58.

Wassermann, R. (2000). Resource Bounded Belief Revision. Ph.D. thesis, University of Amsterdam. 